\title{
The polyhedral nature of LINERs: an XMM-Newton view of LINERs in radio galaxies
}

\author{
M. Gliozzi ${ }^{1}$, L. Foschini ${ }^{2}$, R. M. Sambruna ${ }^{3}$, and F. Tavecchio ${ }^{4}$
}

\author{
${ }^{1}$ George Mason University, 4400 University Drive, Fairfax, VA 22030, USA \\ e-mail: mario@physics.gmu.edu \\ 2 INAF/IASF-Bologna, via Gobetti 101, 40129 Bologna, Italy \\ 3 NASA Goddard Space Flight Center, Code 661, Greenbelt, MD 20771, USA \\ ${ }^{4}$ INAF/Osservatorio Astronomico di Brera, via Bianchi 46, Merate and via Brera 28, Milano, Italy
}

Received 3 August 2007 / Accepted 19 November 2007

\begin{abstract}
Aims. We investigate the origin of X-rays and the nature of accretion flow in 4 low-ionization nuclear emission-line regions (LINERs) hosted by radio galaxies, namely NGC 1692, PKS 0625-35, 3C 88, 3C 444, recently observed with XMM-Newton.

Methods. We combine the results from the time-averaged spectral analysis with model-independent information from X-ray temporal and spectral variability analyses, and with additional broadband information (specifically from the UV band, covered by the Optical Monitor aboard XMM-Newton, and from archival radio data).

Results. The values of the Eddington ratios $L_{\mathrm{bol}} / L_{\mathrm{Edd}}$ of our sample span 2 orders of magnitude ranging between $\sim 1 \times 10^{-5}$ and $1 \times 10^{-3}$. The 4 AGN are adequately fitted by the same continuum model that comprises at least one thermal component $(k T \sim 0.65-$ $1.45 \mathrm{keV}$ ) and a partially absorbed power law, whose relative contribution and photon index vary substantially from source to source. NGC 1692 and PKS 0625-35 have fairly steep power-law components $(\Gamma \sim 2.5-2.9)$, perhaps indicative of synchrotron emission from the base of a jet. Conversely, the flat photon index derived for $3 \mathrm{C} 88(\Gamma \sim 1.1)$ may be indicative of a heavily absorbed object. Finally, the time-averaged spectral properties of $3 \mathrm{C} 444(\Gamma \sim 1.9$ and an apparent line-like excess around $6.7 \mathrm{keV})$ are more in line with Seyfert-like objects. The temporal analysis reveals that PKS 0625-35 and 3C 88 are significantly variable in the soft $(0.2-1 \mathrm{keV})$ energy band. PKS 0625-35 also shows suggestive evidence of spectral variability on timescales of months, with a spectral softening associated with the source brightening. NGC 1692 is only marginally variable in the soft band, whereas 3C 444 does not show significant variability on short timescales. The main findings from the broadband analysis can be summarized as follows: 1) 3C 444, PKS 0625-35, and NGC 1692 have $\alpha_{\mathrm{OX}}$ values consistent with the $\alpha_{\mathrm{OX}}-l_{\mathrm{UV}}$ correlation found by Steffen et al. (2006, AJ, 131, 2826) for Seyfert-like objects. 2) No positive correlation is found between $L_{X}$ and the inclination angle, suggesting that the X-ray emission is not beamed. 3) The values of the radio-loudness are inversely proportional to the Eddington ratio and locate our objects in between the "radio-loud" and "radio-quiet" branches in the $R-l_{\mathrm{Uv}}$ plane proposed by Maoz (2007, MNRAS, 377, 1696).
\end{abstract}

Key words. galaxies: active - galaxies: nuclei - X-rays: galaxies - radio continuum: galaxies

\section{Introduction}

The recent discovery of $H S T$ and ground-based observations that most nearby galaxies harbor supermassive black holes (e.g., Magorrian et al. 1998; Gebhardt et al. 2003), provides convincing evidence that active galactic nuclei (AGN) and normal galaxies are fundamentally connected. It is now generally accepted that there is not a sharp distinction between AGN and normal galaxies, but rather a continuous distribution of activity levels between the two extremes. Therefore, of crucial importance is the role played by low-power AGN, because they may represent the link between powerful AGN and normal galaxies.

The relevance of low-power AGN is bolstered by the finding that they make up a very substantial fraction of the local galaxy population. Indeed, according to the Palomar survey (Ho et al. 1997), over $40 \%$ of nearby galaxies contain low-power AGN, and are optically classified as low-ionization nuclear emissionline regions (LINERs) or as "transition objects", i.e. objects with optical spectra intermediate between those of pure LINERs and H II regions (Heckman 1980).

In the past, several studies investigated the nature of the ionizing mechanism that produces LINER-like optical spectra (i.e.,
$[\mathrm{O} \mathrm{II}] /[\mathrm{O} \mathrm{III}]>1$ and $[\mathrm{O} \mathrm{I}] /[\mathrm{O} \mathrm{III}]>0.33)$ and yielded different viable solutions. For example, Heckman (1980) and Dopita \& Sutherland (1995) proposed shock-heated gas as main ionizing mechanism. Alternatively, the ionization can be provided by very hot stars (such as Wolf-Rayet stars) clustered around the host galaxy nucleus (e.g., Terlevich \& Melnick 1985). Finally, it has been proposed that LINERs can be powered by low-power AGN (e.g., Filippenko \& Halpern 1984).

Currently, the dominant ionization mechanism responsible for these unusual line ratios is still a matter of debate. Nevertheless, it is generally accepted that a sizable fraction of LINERs is associated with AGN. This conclusion is supported by the discovery of broad emission lines in many LINER spectra (e.g., Eracleous \& Halpern 2001), by X-ray imaging and spectral studies (e.g., Terashima \& Wilson 2003; Dudik et al. 2005), and by the fact that LINERs are often associated with compact radio sources with high brightness temperatures (Nagar et al. 2005).

However, the nature of the AGN producing LINER-like spectra is still controversial. For example, based on the study of non-simultaneous spectral energy distributions (SEDs) of seven LINERs, Ho (1999) outlines the lack of a "big blue bump" (usually interpreted as signature for standard efficient accretion 
Table 1. Source properties.

\begin{tabular}{llcccccc}
\hline \hline Source & Class & $z$ & $\begin{array}{c}N_{\mathrm{H}, \mathrm{Gal}} \\
{\left[10^{20} \mathrm{~cm}^{-2}\right]}\end{array}$ & $\begin{array}{c}\log M_{\mathrm{BH}}^{a} \\
{\left[M_{\odot}\right]}\end{array}$ & $\begin{array}{c}S_{\text {core,5GHz }}^{b} \\
{[\mathrm{Jy}]}\end{array}$ & $\begin{array}{c}S_{\text {tot,5GHz }}^{b} \\
{[\mathrm{Jy}]}\end{array}$ & $\begin{array}{c}\mathfrak{R}^{b} \\
{\left[S_{\text {core }} / S_{\text {ext }}\right]}\end{array}$ \\
\hline NGC 1692 & FRI & 0.035 & 3.54 & 9.01 & 0.04 & 1.81 & 0.023 \\
PKS 0625-35 & FRI & 0.055 & 7.08 & 9.19 & 0.60 & 2.12 & 0.395 \\
3C 88 & FRI/FRII & 0.030 & 8.81 & 8.70 & 0.16 & 2.14 & 0.087 \\
3C 444 & FRII & 0.153 & 2.61 & 9.28 & $<0.002$ & 1.99 & $<0.0009$ \\
\hline
\end{tabular}

${ }^{a}$ From Bettoni et al. (2003); the uncertainty is $\sim 0.4$ dex. ${ }^{b}$ From Morganti et al. (1993).

disks), arguing that LINERs have intrinsically different SEDs compared to "normal" AGN and that they are likely hosts of radiatively inefficient accretion flows (RIAFs). On the other hand, Satyapal et al. (2004) combining mid-IR spectroscopy with X-ray imaging conclude that LINERs are often heavily absorbed and that the intrinsic SEDs in LINERs are not necessarily different from those of standard AGN. This conclusion finds support from the recent findings of Maoz (2007): using highquality UV data for a sample of 13 unabsorbed LINERs observed with $H S T$, Maoz argues that the SEDs of low-luminosity AGN (LLAGN) are similar to those of Seyfert galaxies.

Previous X-ray studies of low-power AGN with Chandra demonstrate the importance of X-rays to make progress in this field (e.g., Di Matteo et al. 2001, 2003; Pellegrini et al. 2005; Evans et al. 2006; Balmaverde et al. 2006). However, the nature of the central engine in low-power AGN is still a matter of debate, with some authors favoring a jet-dominated scenario and others an accretion-dominated scenario, which in turn might be either radiatively efficient or inefficient. One of the reasons for this controversy is the poor discriminating power of the low signal-to-noise $(\mathrm{S} / \mathrm{N})$ spectra, which do not allow one to choose between the competing scenarios.

In order to break this spectral degeneracy, higher $\mathrm{S} / \mathrm{N}$ spectra combined with additional model-independent constraints are required. This is the approach that we (Gliozzi et al. 2003) adopted to investigate the origin of the X-rays in the nuclear region of the nearby FRI NGC 4261, which is classified as Weak-Line Radio Galaxy (WLRG) and LINER (Lewis et al. 2003). By definition, WLRGs are radio galaxies with very weak [O III] emission: $E W([\mathrm{O} \mathrm{III}])<10 \AA$ (Tadhunter et al. 1998). However, no formal constraints are placed on the optical line ratios, therefore, an object classified as WLRG is not necessarily a LINER and vice versa. Nevertheless, a systematic study of the optical properties of a sample WLRGs has shown that the majority of the objects can be robustly classified as LINERs (Lewis et al. 2003). For the sake of simplicity, in the following we will use the term WLRGs to indicate radio galaxies with weak optical lines, which are optically classified as LINERs.

Exploiting the superior sensitivity of XMM-Newton, in NGC 4261 we found evidence for significant temporal and spectral variability on short timescales, and the presence of an unresolved Fe K line at $\sim 6.9 \mathrm{keV}$ of nuclear origin. We thus concluded that the inner jet is unlikely to dominate the X-ray emission. Similar conclusions were reached by Rinn et al. (2005), using Chandra, XMM-Newton, and BeppoSAX archival data of 9 WLRGs. Another relevant result from Rinn et al. (2005) is the detection of intrinsic absorption in 6 out 9 objects, with 4 sources having $N_{\mathrm{H}}>10^{22} \mathrm{~cm}^{-2}$. This is in contrast with the systematic lack of absorption found in a larger sample of non-LINER FRIs observed with Chandra or XMM-Newton (Donato et al. 2004), and suggests that WLRGs may form a distinct class compared to other low-power radio galaxies.

To summarize, there is still no clear consensus on what is the dominant source of X-rays in low-power AGN/LINERs and what is the nature of accretion flow onto their black holes. Are the different conclusions just a consequence of the lower photon statistics, or are they reflecting intrinsic differences among objects of the same class? And if so, are these differences related to the nuclear power and/or to the beaming of the sources? To answer these questions and make progress in the study of accretion in AGN, it is important to expand the sample of low-power radio galaxies with high-quality X-ray data.

Here, we investigate the nature of 4 WLRGs (optically classified as LINERs) NGC 1692, PKS 0625-35, 3C 88, and 3C 444, whose properties are described in Sect. 2 and summarized in Table 1. Specifically, using observations from XMM-Newton (described in Sect. 3), we try to shed light on the origin of $\mathrm{X}$-rays and the nature of the accretion mechanism. To this end, we combine several pieces of information obtained from the time-averaged X-ray spectroscopy (discussed in Sect. 4), from the X-ray variability (described in Sect. 5), and from broadband analysis, which combines the simultaneous X-ray/UV coverage with archival radio data (in Sect. 6). Hereafter, we adopt $H_{0}=71 \mathrm{~km} \mathrm{~s}^{-1} \mathrm{Mpc}^{-1}, \Omega_{\Lambda}=0.73$ and $\Omega_{\mathrm{M}}=0.27$ (Bennet et al. 2003).

\section{Sample properties}

The 4 targets, whose properties are reported in Table 1, were selected from a sample of 20 WLRGs recently studied in the optical range by Lewis et al. (2003). This sample was in turn drawn from the sample of WLRGs presented by Tadhunter et al. (1998). The main characteristics of the 4 radio galaxies can be summarized as follows:

- they all contain a confirmed LINER on the basis of measurements of optical line ratios (Lewis et al. 2003);

- two (NGC 1692 and PKS 0625-35) are FRI and two (3C 88 and $3 \mathrm{C} 444)$ FRII galaxies, although the classification of 3C 88 is only morphological. According to the optical criteria proposed by Jackson \& Rawlings (1997), the two FRII galaxies are classified as low-excitation radio galaxies (LEGs);

- they have been previously detected at X-rays in the ROSAT all sky survey; PKS 0625-35 was also observed with BeppoSAX (Trussoni et al. 1999). The X-ray luminosities span two orders of magnitude; 
Table 2. Observation log.

\begin{tabular}{lccc}
\hline \hline Source & $\begin{array}{c}\text { Date } \\
{[\mathrm{dd} / \mathrm{mm} / \text { yyyy }]}\end{array}$ & $\begin{array}{c}\text { MOS exposure } \\
{[\mathrm{ks}]}\end{array}$ & $\begin{array}{c}\text { MOS rate }^{a} \\
{\left[\mathrm{~s}^{-1}\right]}\end{array}$ \\
\hline NGC 1692 & $15 / 08 / 2005$ & 13.8 & $(6.7 \pm 0.2) \times 10^{-2}$ \\
PKS 0625-35 & $03 / 05 / 2005$ & 1.5 & $(7.4 \pm 0.2) \times 10^{-1}$ \\
& $27 / 08 / 2005$ & 13.1 & $(8.1 \pm 0.1) \times 10^{-1}$ \\
3C 88 & $02 / 09 / 2006$ & 22.4 & $(3.4 \pm 0.2) \times 10^{-2}$ \\
3C 444 & $02 / 11 / 2005$ & 18.1 & $(1.2 \pm 0.1) \times 10^{-1}$ \\
\hline
\end{tabular}

${ }^{a}$ The MOS1 count rate is consistent with the MOS2 value within the quoted errors, whereas the pn count rate is $\sim 2.5$ times the MOS count rate.

- they are all nearby galaxies $(z=0.03-0.15)$ well studied at longer wavelengths, especially in the radio band with ATCA and VLA observations;

- the masses of their supermassive black holes have been estimated in an homogeneous way by Bettoni et al. (2003).

In summary, although the radio core fluxes and the morphological classification are different, the 4 sources share several fundamental common properties: 1) optically, they are all formally classified as LINERs; 2) in the radio band, they have similar extended fluxes, which, unlike the core fluxes (possibly affected by beaming), are generally considered reliable indicators of the source intrinsic power; 3) they have similar black hole masses, which are indistinguishable from each other when their uncertainty of the order of 0.4 dex is accounted for; 4) recent radio, optical, and X-ray studies of the core properties of radio galaxies have suggested that FRII/LEGs are indeed unified with FRI galaxies (e.g., Chiaberge et al. 2002; Hardcastle et al. 2006).

\section{Observations and data reduction}

We observed our 4 targets with XMM-Newton between August, 2005 and September, 2006. The nominal durations range between 15 and $35 \mathrm{ks}$. All of the EPIC cameras (Strüder et al. 2001; Turner et al. 2001) were operated in full-frame mode with thin or medium filters, depending on the presence of bright nearby sources in the field of view. As a precaution, for PKS 062535 and 3C 444 the MOS cameras were operated in small window mode to prevent photon pile-up. The recorded events were screened to remove known hot pixels and other data flagged as bad; only data with FLAG $=\mathbb{0}$ were used.

The data were processed using the latest CCD gain values. For the temporal analysis, events corresponding to pattern 012 (singles, doubles, triples, and quadruples) in the MOS cameras and 0-4 (singles and doubles only, since the pn pixels are larger) in the pn camera were accepted. For the spectral analysis, only single events were considered in order to exploit their better calibration down to $0.2 \mathrm{keV}$ (Kirsch 2006). Arf and rmf files were created with the XMM-Newton Science Analysis Software (SAS) 7.0. Investigation of the full-field light curves revealed the presence of one period of background flaring for NGC 1692, and several flares for the EPIC pn observation of $3 \mathrm{C} 88$. These events were excluded, reducing the effective total exposures time to the values reported in Table 2. The extraction radii used for source spectra and light curves range between 20" (for 3C 88) and $40^{\prime \prime}$ (for 3C 444), depending on the extension of the source and its proximity to the CCD edges. For reference, with the chosen cosmological parameters, $10^{\prime \prime}$ correspond to $6.9 \mathrm{kpc}$ for NGC 1692, $10.5 \mathrm{kpc}$ for PKS 0625-35, $5.9 \mathrm{kpc}$ for 3C 88, and $26.3 \mathrm{kpc}$ for $3 \mathrm{C} 444$. Background spectra and light curves were extracted from source-free circular regions on the same chip as the source, with extraction radii $\sim 2$ times larger than those used for the source. There are no signs of pile-up in the pn or MOS cameras according to the SAS task epatplot. The RGS data have signal-to-noise ratio $(S / N)$ that is too low for a meaningful analysis.

The observation log with dates of the observations, EPIC net exposures, and the average count rates are reported in Table 2.

The spectral analysis was performed using the XSPEC v.12 . 3.0 software package (Arnaud 1996; Dorman \& Arnaud 2001). The EPIC data have been re-binned in order to contain at least 20 counts per channel, depending on the brightness of the source. The errors on spectral parameters are at $90 \%$ confidence level for one interesting parameter $\left(\Delta \chi^{2}=2.71\right)$.

The data from the OM (Mason et al. 2001) were processed with the latest calibration files using the SAS task omichain, which provides count rates in the $U(3440 \AA)$, UVW1 (2910 ̊), and UVM2 (2310 ̊) bands. The count rates were then converted into magnitudes using the formula mag $=-2.5 \log$ (count rate) + zero-point (where the zero-points are 18.259, 17.204, and 15.772 , for the $U, \mathrm{UVW} 1$, and UVM2 bands, respectively), and corrected for systematics. The values were corrected for extinction (see Sect. 6 for more details) and converted to fluxes by using standard formulae (e.g., Zombeck 1990).

\section{X-ray spectral analysis}

The X-ray spectra represent one of the most used and effective tools to investigate the accretion process in AGN. In the following, we perform a time-averaged spectral analysis of the EPIC data in the $0.2-10 \mathrm{keV}$ energy range. For the analysis of the continuum, the spectra from the three EPIC cameras (pn, MOS1, and MOS2) have been combined to improve the overall S/N. The EPIC pn alone was used for the $\mathrm{Fe} \mathrm{K} \alpha$ analysis in virtue of its larger throughput and effective area above $6 \mathrm{keV}$. For the sake of clarity, in the figures describing the results from the spectral analysis we show only the EPIC pn data.

\subsection{Continuum}

The background-subtracted EPIC spectra in the $0.2-10 \mathrm{keV}$ energy range were fitted with models of increasing complexity until an adequate fit was achieved. The significance of the different spectral components was obtained from an $F$-test. As an operative rule, if the addition of a spectral component improves the fit by $\Delta \chi^{2}$ such that the associated $P_{F} \leq 0.05$, the new component is considered significant.

In general, simple power-law models, modified by Galactic interstellar absorption, do not provide a good representation of the XMM-Newton EPIC spectra. Figure 1 shows the data-tomodel ratios for our 4 targets. Large residuals at soft energies are present in NGC 1692, 3C 88, and 3C 444, suggesting the presence of additional (thermal) components. The residuals of 3C 444 also suggest the possible presence of a line-like feature around 6-7 keV. Only PKS 0625-35 is apparently well described by a simple power law. However, a more detailed analysis indicates that more complex spectral models provide better fits also for this source (see below).

The baseline model able to fit the spectra of the 4 targets requires at least 2 components: 1 ) a thermal component, described 

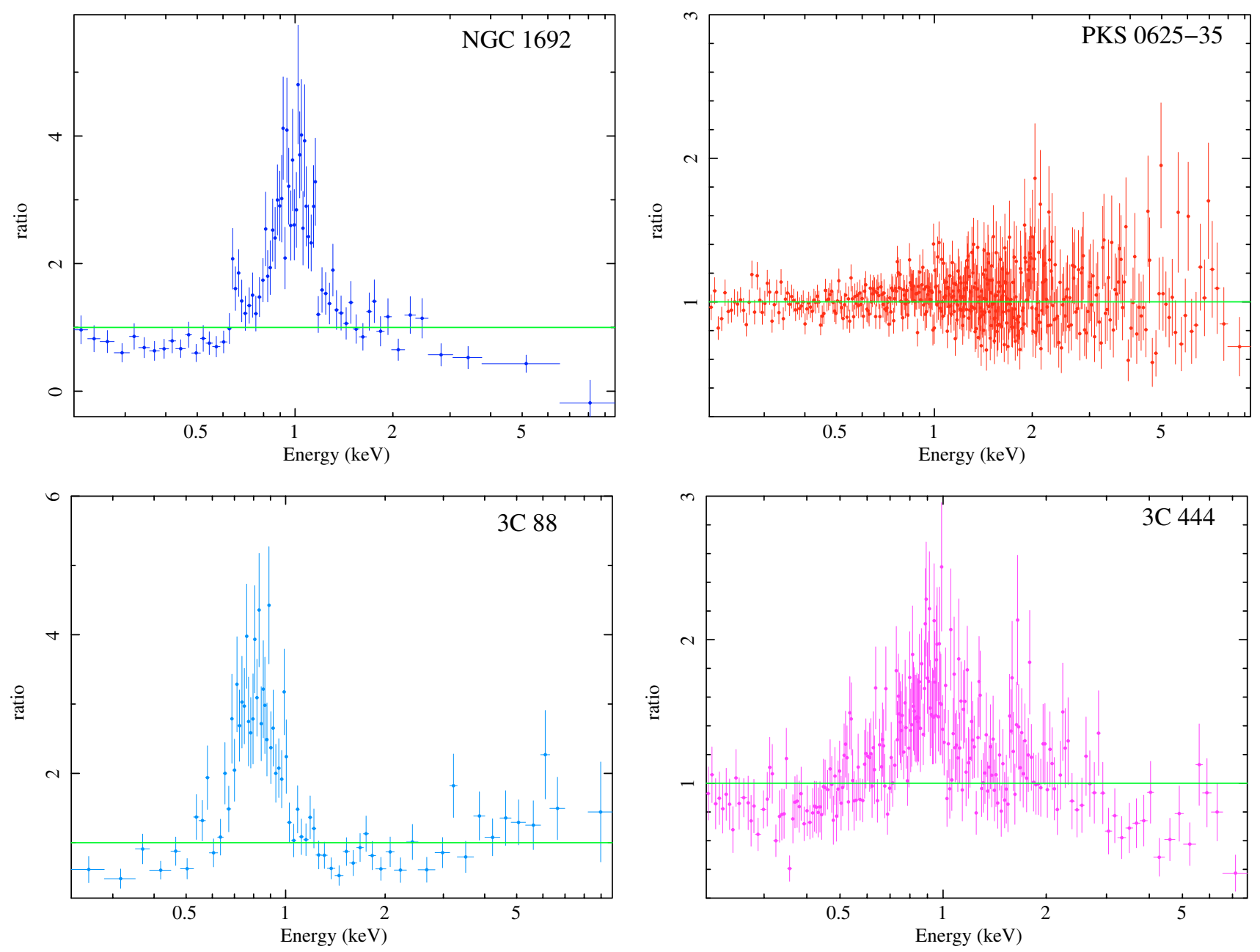

Fig. 1. Data-to-model ratio obtained by fitting the EPIC pn spectra in the $0.2-10 \mathrm{keV}$ energy range with a simple power-law model absorbed by Galactic $N_{\mathrm{H}}$. Large residuals at soft energies are present in NGC 1692, 3C 88, and 3C 444, which also shows an excess around $6 \mathrm{keV}$, PKS 0625-35 is apparently adequately described by a simple power law. However, a more detailed analysis suggests more complex spectral models also for this source (see text for details).

by apec in XSPEC (Smith et al. 2001), which parameterizes the emission from a collisionally-ionized plasma and is usually associated with a diffuse circum-nuclear component that is frequently detected in radio galaxies by Chandra thanks to its subarcsecond spatial resolution (e.g., Donato et al. 2004), and 2) a partially absorbed power law that emerges at harder energies and parameterizes the emission from the unresolved nuclear component. The metal abundances of the thermal model were initially left free to vary between 0.2 and 1.2 solar values, and then fixed at the best fit value. Also the covering factor in the partial absorption model (zpcfabs in XSPEC) was kept fixed at the best fit value.

We have also tried alternative models such as broken powerlaw (hereafter bkn) or double power-law models, with one absorbed power law with $\Gamma$ fixed at 1.8 and the other power law unabsorbed. However, none of the alternative models yielded better (or statistically equivalent) fits compared to results from the baseline model described above. In the case of the double power-law model, the spectral parameters are in general poorly constrained and often the contribution of one of the two power laws becomes negligible. The only exception is PKS 062535 , for which a broken power law $\left(\Gamma_{1}=2.35 \pm 0.06, E_{\mathrm{br}}=\right.$ $0.8 \pm 0.3 \mathrm{keV}, \Gamma_{2}=2.54 \pm 0.03$ ) provides an equally good statistical fit using less free parameters than the baseline model $\left(\chi^{2}=790.1\right.$ for 765 d.o.f. and $\chi^{2}=791.3$ for 762 d.o.f., for bkn and the baseline model, respectively). However, since the fluxes and luminosities are fully consistent with those obtained using the partially absorbed model, for consistency and to allow a direct comparison with the other sources, in Table 3 we report the results of the spectral fitting of the continua (and the significance of the individual components) of the baseline model described above.

The photon indices obtained for our sample vary substantially from source to source, ranging from $\Gamma \sim 1.1$ for $3 \mathrm{C} 88$ to $\Gamma \sim 2.5-2.9$ for PKS $0625-35$ and NGC 1692 respectively, with 3C 444 being the only one to show a typical Seyfert-like $\Gamma \sim 1.9$. All the sources statistically require at least one thermal component with $k T$ in the range $0.65-1.45 \mathrm{keV}$.

Although, the baseline model is the same for the 4 sources, the contribution of the different components changes significantly for the different objects, as it is clearly shown in Fig. 2, where the deconvolved $E F_{E}$ spectra are plotted. In addition, NGC 1692 statistically requires the presence of a second thermal component.

One of the goals of the X-ray spectral analysis is to investigate the presence of intrinsic absorption. Table 3 already indicates that there is evidence for moderate absorption $\left(N_{\mathrm{H}} \sim\right.$ $10^{21} \mathrm{~cm}^{-2}$ ) in NGC 1692, PKS 0625-35, and 3C 444, whereas in $3 \mathrm{C} 88$ a local absorber is not statistically required. However, the latter result might be hampered by the limited photon 
Table 3. X-ray continuum spectral properties.

\begin{tabular}{lccccccc}
\hline \hline Source & \multicolumn{2}{c}{ Thermal component } & \multicolumn{3}{c}{ Partial absorption } & Power law & \multirow{2}{*}{$\chi^{2}$ (d.o.f.) } \\
& $k T$ & $P_{F}^{a}$ & $N_{\mathrm{H}}$ & $C v r$ & $P_{F}^{a}$ & $\Gamma$ & \\
& {$[\mathrm{keV}]$} & {$[\%]$} & {$\left[10^{21} \mathrm{~cm}^{-2}\right]$} & {$[\%]$} & {$[\%]$} & & \\
\hline NGC 1692 & $0.79 \pm 0.08$ & $3 \times 10^{-9}$ & $6_{-4}^{+12}$ & 95 & $2 \times 10^{-2}$ & $2.9_{-1.1}^{+0.4}$ & $131.6(143)$ \\
PKS 0625-35 & $0.64 \pm 0.19$ & $3 \times 10^{-3}$ & $1.5_{-1.1}^{+1.8}$ & 12 & $1 \times 10^{-2}$ & $2.52_{-0.03}^{+0.02}$ & $791.3(762)$ \\
3C 88 & $0.64 \pm 0.02$ & $1 \times 10^{-15}$ & $0.5_{-0.5}^{+6.2}$ & 50 & $5 \times 10^{-1}$ & $1.11_{-0.14}^{+0.18}$ & $164.6(133)$ \\
3 C 444 & $1.45 \pm 0.15$ & $7 \times 10^{-7}$ & $1.2_{-0.5}^{+0.7}$ & 95 & $6 \times 10^{-5}$ & $1.92_{-0.33}^{+0.27}$ & $394.4(377)$ \\
\hline
\end{tabular}

${ }^{a}$ Probability associated with the $F$ statistic (the smaller the value the higher the significance of the spectral component). NGC 1692 requires a second thermal component with $k T=1.44 \pm 0.15\left(P_{F}=5 \times 10^{-9}\right)$. The quoted uncertainties correspond to $90 \%$ confidence levels.
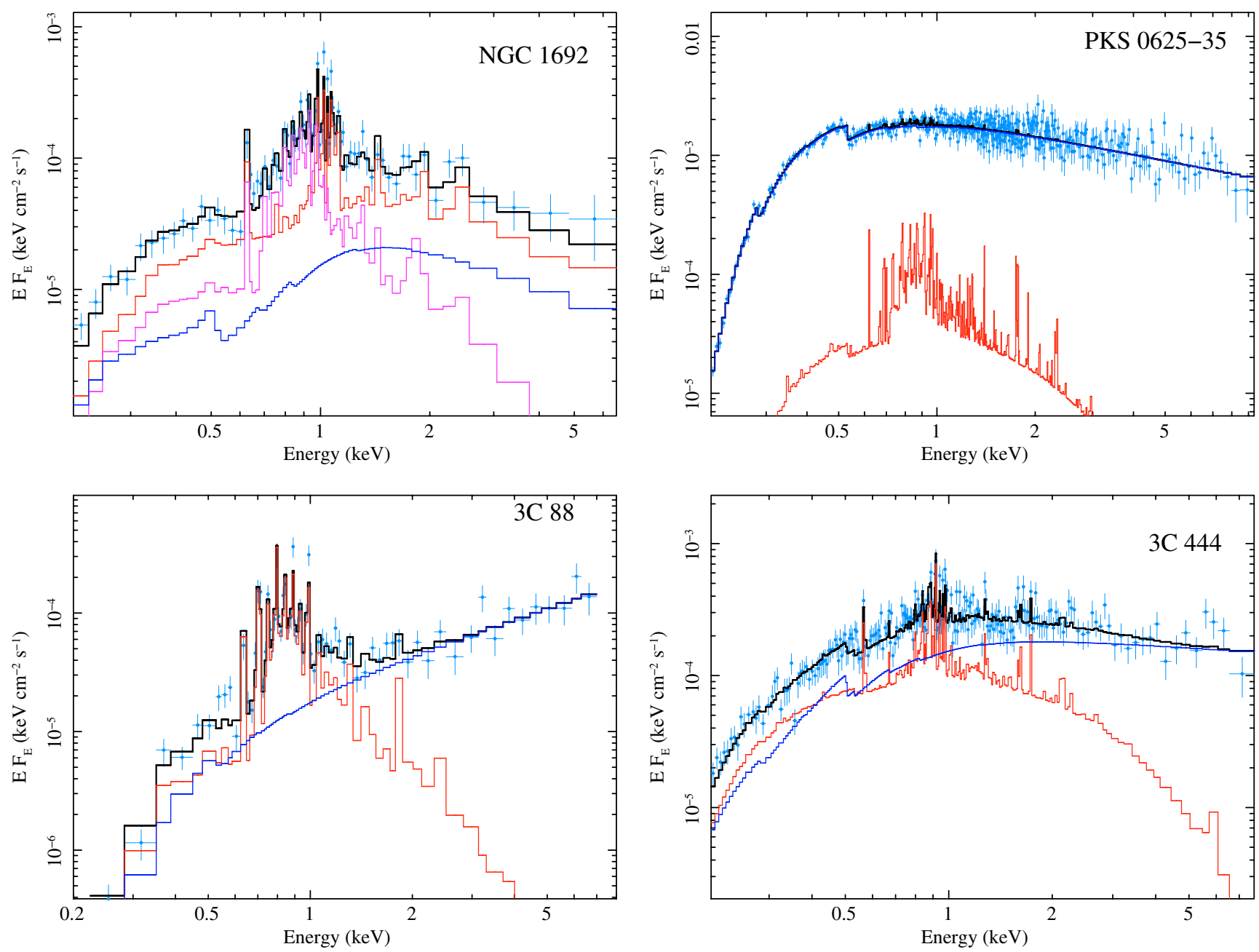

Fig. 2. Deconvolved EPIC pn spectra in the $0.2-10 \mathrm{keV}$ energy range, fitted with a partially absorbed power-law plus a thermal component. NGC 1692 requires an additional thermal component, whereas 3C 444 shows a line-like excess around $\sim 6-7 \mathrm{keV}$. All models are absorbed by Galactic $N_{\mathrm{H}}$.

statistics, since the unusually flat photon index may indicate that the X-ray spectrum of $3 \mathrm{C} 88$ is affected by substantial absorption. Indeed, if $\Gamma$ is fixed at a more reasonable value of 1.4 , the resulting fit $\left(\chi^{2}=168.6\right.$ for 134 d.o.f.) is still comparable to the one obtained with $\Gamma$ free, however, the resulting intrinsic absorption, $N_{\mathrm{H}}=1.8_{-1.4}^{+3.7} \times 10^{22} \mathrm{~cm}^{-2}$, is now substantial.

In order to test whether there is degeneracy between $\Gamma$ and $N_{\mathrm{H}}$, and to better visualize the relative uncertainties on these parameters, in Fig. 3 we have plotted the confidence contours of
$\Gamma$ and $N_{\mathrm{H}}$. For the sources with the highest count rate, PKS 062535 and $3 \mathrm{C} 444, N_{\mathrm{H}}$ is well constrained, whereas the plots of NGC 1692 and 3C 88 show large uncertainties, which do not allow one to rule out a heavily absorbed scenario.

In Table 4 we report the values for the soft $(0.2-2 \mathrm{keV})$ and hard $(2-10 \mathrm{keV})$ fluxes and luminosities as well as the relative contributions of the power-law component in these energy bands. In general, the hard energy band is dominated by the power-law component with contributions larger than $\sim 80 \%$, 

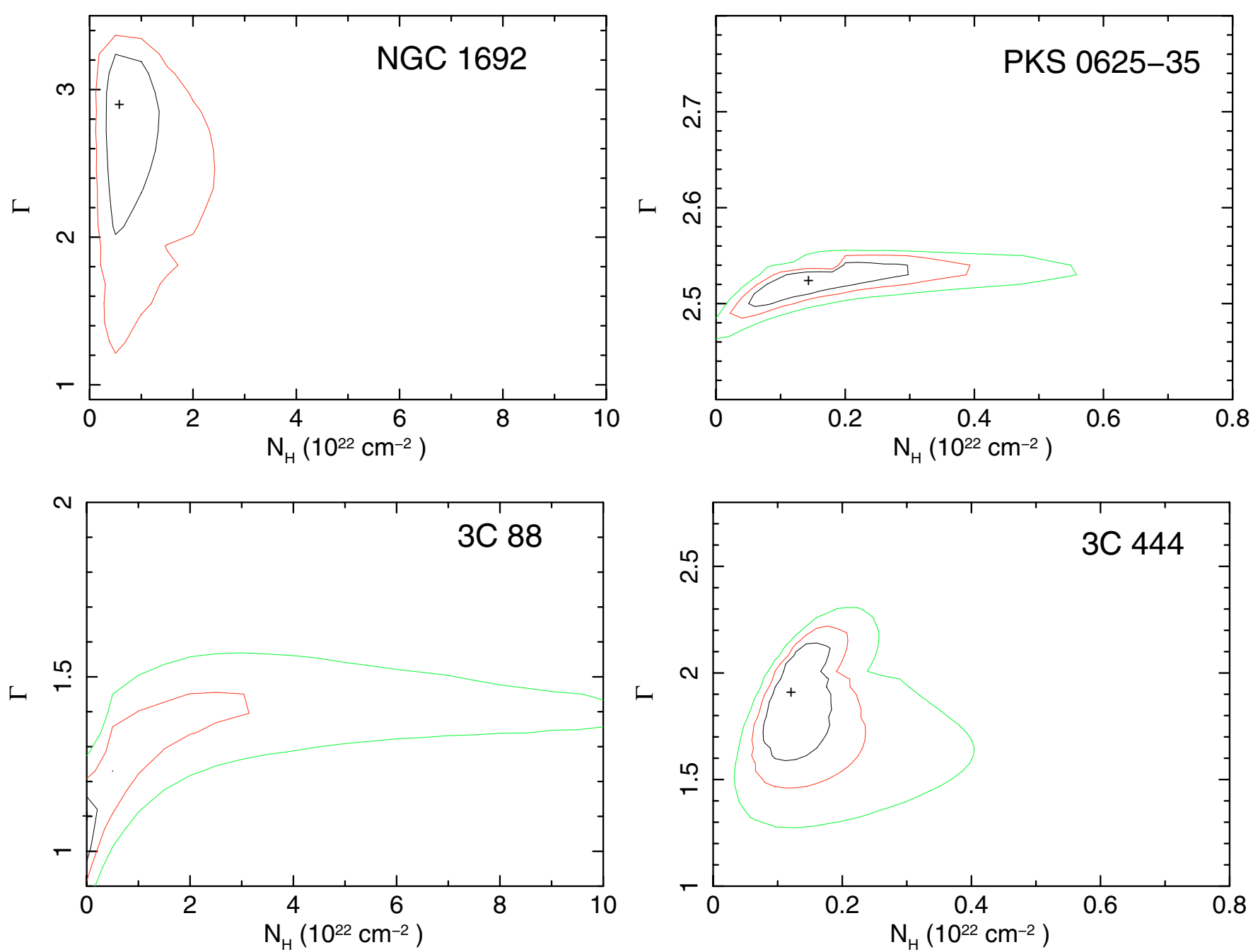

Fig. 3. Confidence contours $(68 \%, 90 \%$, and $99 \%)$ in the $\Gamma-N_{\mathrm{H}}$ plane; for NGC 1692 , only $68 \%$ and $90 \%$ levels are shown. The spectral model used comprises a thermal component and a partially absorbed power law.

Table 4. X-ray fluxes and luminosities.

\begin{tabular}{|c|c|c|c|c|c|c|c|}
\hline Source & $\begin{array}{c}F_{0.2-2 \mathrm{keV}} \\
{\left[\mathrm{erg} \mathrm{cm}^{-2} \mathrm{~s}^{-1}\right]}\end{array}$ & $\begin{array}{l}L_{0.3-2} \mathrm{ke} \\
{\left[10^{42} \mathrm{erg} \mathrm{s}^{-1}\right]}\end{array}$ & $\% L_{\mathrm{PL}}^{a}$ & $\begin{array}{c}F_{2-10 \mathrm{keV}} \\
{\left[\mathrm{erg} \mathrm{cm}^{-2} \mathrm{~s}^{-1}\right]}\end{array}$ & 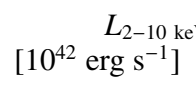 & $\% L_{\mathrm{PL}}^{a}$ & $L_{\mathrm{bol}} / L_{\mathrm{Edc}}^{b}$ \\
\hline NGC 1692 & $2.7 \times 10^{-13}$ & 0.9 & $55 \%$ & $0.9 \times 10^{-13}$ & 0.2 & $39 \%$ & $1 \times 10^{-5}$ \\
\hline PKS 0625-35 & $4.7 \times 10^{-12}$ & 78.2 & $98 \%$ & $2.6 \times 10^{-12}$ & 18.7 & $100 \%$ & $2 \times 10^{-3}$ \\
\hline $3 C 88$ & $1.4 \times 10^{-13}$ & 0.4 & $34 \%$ & $2.8 \times 10^{-13}$ & 0.5 & $99 \%$ & $2 \times 10^{-4}$ \\
\hline $3 \mathrm{C} 444$ & $6.8 \times 10^{-13}$ & 63.0 & $50 \%$ & $5.1 \times 10^{-13}$ & 33.1 & $78 \%$ & $3 \times 10^{-3}$ \\
\hline
\end{tabular}

${ }^{a}$ Percentage of the soft (hard) X-ray luminosity associated with the power-law component. ${ }^{b}$ Eddington ratio $L_{\mathrm{bol}} / L_{\mathrm{Edd}}$, where $L_{\mathrm{bol}}$ was obtained by multiplying the $2-10 \mathrm{keV}$ X-ray luminosity, associated with the power law, by a factor 20 (see text for details).

the only exception being NGC 1692, where the power law contributes only $\sim 40 \%$ of the total $L_{2-10} \mathrm{keV}$.

Using the estimates of the black hole masses reported in Table 1 and the hard X-ray luminosities associated with the power law (hereafter $L_{\mathrm{X}}$ ), we have computed the luminosity ratio $L_{\mathrm{X}} / L_{\mathrm{Edd}}$ (where $L_{\mathrm{Edd}}$ is the Eddington luminosity). Detailed modeling of broad-band SEDs of weak AGN and Galactic black holes in the low/hard state showed that the origin of X-rays (accretion-dominated versus jet-dominated) may indeed depend upon the value of $L_{\mathrm{X}} / L_{\mathrm{Edd}}$, with values below a threshold of
$10^{-6}$ generally associated with a jet-dominated scenario (see $\mathrm{Wu}$ et al. 2007, and references therein for more details). In this framework, the relatively high values of $L_{\mathrm{X}} / L_{\mathrm{Edd}}$ derived for PKS 0625-35 $\left(1 \times 10^{-4}\right), 3$ C $88\left(1 \times 10^{-5}\right)$, and 3C $444\left(1 \times 10^{-4}\right)$ apparently favor an accretion-dominated origin, whereas the relatively low value found for NGC $1692\left(8 \times 10^{-7}\right)$ is more in line with a jet origin. However, one must bear in mind that the above criterion is strongly model-dependent and hence these conclusions should be taken with caution. 
Table 5. Fe K $\alpha$ Gaussian model parameters (rest frame).

\begin{tabular}{lclccc}
\hline \hline Source & $\begin{array}{c}E_{\mathrm{Fe}} \\
(\mathrm{keV})\end{array}$ & $\begin{array}{l}\sigma_{\mathrm{Fe}} \\
(\mathrm{keV})\end{array}$ & $\begin{array}{c}I_{\mathrm{Fe}} \\
\left(10^{-6} \mathrm{ph} \mathrm{cm}^{-2} \mathrm{~s}^{-1}\right)\end{array}$ & $\begin{array}{c}E W \\
(\mathrm{keV})\end{array}$ & $\Delta \chi^{2}{\text { (d.o.f. })^{a}}^{+2.4}$ \\
\hline PKS 0625-35 & 6.4 & 0.01 & $1.5_{-1.5}^{+2.4}$ & $0.079(<0.182)$ & $-1.3(1)$ \\
3C 88 & 6.4 & 0.01 & $1.5_{-1.5}^{+5.5}$ & $0.435(<0.643)$ & $-1.5(1)$ \\
$3 \mathrm{C} 444$ & $6.7_{-0.4}^{+0.4}$ & $0.35_{-0.35}^{+0.47}$ & $4.4_{-2.7}^{+2.8}$ & $0.550_{-0.380}^{+0.690}$ & $-5.7(3)$ \\
\hline
\end{tabular}

${ }^{a}$ Decrease in the $\chi^{2}$ (and additional number of degrees of freedom) obtained by adding a Gaussian line to the best fit continuum.

In general, the accretion efficiency in black hole systems is assessed by computing the Eddington ratio $L_{\mathrm{bol}} / L_{\mathrm{Edd}}$, which requires the use of a bolometric correction factor. Often in AGN studies this factor has been computed by assuming a mean energy distribution, which cannot be accurate for all AGN (Elvis et al. 1994). A further problem with this approach is that the contribution from the IR bump is included in the computation of the bolometric luminosity. However, the IR emission in AGN is generally dominated by reprocessed emission and hence its inclusion would lead to an overestimate of the accretion luminosity. More recently, Marconi et al. (2004) proposed an alternative approach, based on Monte Carlo simulations of a template of AGN SEDs, which yields different bolometric correction factors depending on the AGN X-ray luminosity. Finally, Vasudevan \& Fabian (2007), using actual SEDs from 54 AGN, showed that the dependence on the X-ray luminosity is not so straightforward, although there seems to be a bimodal dependence on the Eddington ratio: for $L_{\text {bol }} / L_{\text {Edd }}<0.1$ the correction factor to $L_{2-10 \mathrm{keV}}$ is $15-25$, while above this threshold the correction factor is $40-70$.

Following Vasudevan \& Fabian (2007) and bearing in mind several caveats (for example, the above results are derived from non-simultaneous SEDs of radio-quiet AGN), we computed the bolometric luminosity for our targets by multiplying $L_{2-10 \mathrm{keV}}$ by a correcting factor of 20 (i.e., we assume that the $2-10 \mathrm{keV}$ luminosity represents $5 \%$ of the bolometric luminosity). The results, reported in the last column of Table 4 , indicate that the Eddington ratio for the 4 targets spans more than two orders of magnitude ranging between $\sim 1 \times 10^{-5}$ and $3 \times 10^{-3}$. While the lowest values (obtained for NGC 1692 and 3C 88) seem to favor a radiatively inefficient accretion flow hypothesis (e.g., Quataert et al. 1999), a radiatively efficient scenario cannot be ruled out a priori for PKS $0625-35$ and 3C 444, if we assume that the bulk of the X-ray emission is produced in the accretion flow. These conclusions hold even if the uncertainties for the black hole masses are accounted for, given that they cause changes of $L_{\text {bol }}$ by a factor of a few and not by orders of magnitude.

\subsection{Iron $\mathrm{K} \alpha$ line}

After finding adequate parameterizations for the spectral continua, we have investigated whether a Fe $\mathrm{K} \alpha$ line could improve the overall fits. An inspection of the residuals does not show evidence for any prominent line-like features in the 6-7 keV range for PKS 0625-35 and 3C 88 (NGC 1692 has no spectral coverage of the interesting energy range for this analysis). Nevertheless, we have added a narrow ( $\sigma=0.01 \mathrm{keV})$ Gaussian line with energy fixed at $6.4 \mathrm{keV}$ (in the source's rest frame) to the best-fit continuum models, in order to estimate the $90 \%$

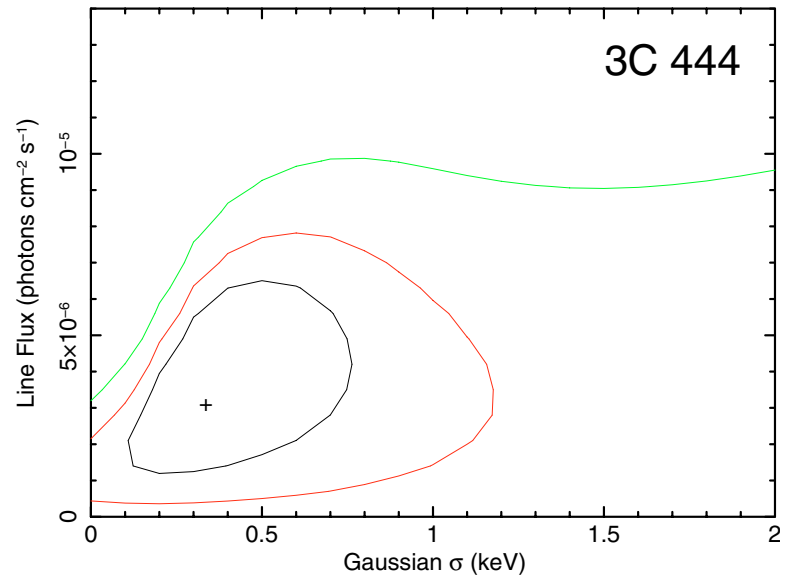

Fig. 4. Confidence contours in the line intensity-line width plane for 3C 444, using a single Gaussian model. The confidence levels correspond to $68 \%, 90 \%$, and $99 \%$.

upper limit on the line contribution, as measured by the equivalent width. The results are summarized in Table 5.

Unlike the other sources, the spectral residuals of 3C 444 around 6-7 keV apparently reveal a relatively broad hump centered around $\sim 6.7 \mathrm{keV}$ (in the source's rest frame). Adding a Gaussian line to the best-fitting continuum model improves the fit of the EPIC pn spectrum only marginally: $\Delta \chi^{2} \sim-6$ for 3 additional parameters (however, if the line energy is kept fixed at $6.7 \mathrm{keV}$, the line significance increases at more than 90\%).

When fitted with a single Gaussian, the line appears to be centered around $6.7 \mathrm{keV}$, possibly broad $(\sigma \sim 0.35 \mathrm{keV})$ and fairly strong $(E W \sim 550 \mathrm{eV})$. Similar results are obtained when a diskline model (with inclination angle fixed at $60^{\circ}$ ) is used instead of a Gaussian line. We have also tried to fit the linelike feature with two Gaussian models, keeping one line energy fixed at $6.4 \mathrm{keV}$ (in the source's rest frame). The overall fit does not improve significantly ( $\Delta \chi^{2}=-1.5$ for 2 additional d.o.f.), and the results, $E_{1}=6.4 \mathrm{keV}, \sigma_{1} \ll 10^{-3} \mathrm{keV}, E W<440 \mathrm{eV}$, $E_{2}=7.0_{-0.7}^{+0.5} \mathrm{keV}, \sigma_{1}=0.2_{-0.2}^{+0.7} \mathrm{keV}, E W=475_{-99}^{+744} \mathrm{eV}$, indicate that the line parameters are poorly constrained.

The contour plots of the line flux and physical width (see Fig. 4) obtained using a single Gaussian model, apparently confirm that the line is detected at more than $90 \%$ level and that it is resolved at more than $68 \%$ confidence level. However, a broader hard X-ray energy range covering the region where the reflection component peaks, and longer observations with at least $10^{4}$ counts in the $2-10 \mathrm{keV}$ energy band are necessary to firmly establish the presence of a broad line and constrain the line parameters (Guainazzi et al. 2006). 


\section{X-ray variability}

Although the time-averaged spectral analysis offers important insights into the physical processes at work in AGN, it cannot be considered an exhaustive diagnostic. This is because the $\mathrm{X}$-ray spectral analysis is based on the comparison of the observed spectrum with a model spectrum (the spectral fit), and hence the results are by definition model-dependent. In addition, the same spectra can often be adequately fitted by different combinations of spectral parameters for a specific model, or by different physical models. This spectral degeneracy severely affects spectra with low $\mathrm{S} / \mathrm{N}$ (either due to the intrinsic faintness of the sources or to a limited sensitivity of the instrument), but also higher quality spectra are not immune from this problem. In order to progress in our understanding of the AGN phenomenon, the results from the X-ray time-averaged spectral analysis need to be validated and complemented by other constraints (possibly obtained in a model-independent way) such as temporal and spectral variability studies. XMM-Newton with its high throughput and highly elliptical orbit is one of the best instruments to investigate the AGN variability on short and medium timescales.

The importance of combining information from the spectral and temporal analyses is underscored by the power of $\mathrm{X}$-ray spectral variability studies in discriminating between jetdominated and accretion-dominated emission.

For example, an X-ray spectrum described by a power law with $\Gamma$ ranging between 1.5 and 2.5 can be equally well interpreted in the framework of jet models (where inverse Compton and synchrotron are the dominant emission processes), or in the framework of coronal models, by varying the geometry configuration of static models (e.g., Haardt et al. 1994) or by assuming a mildly relativistic outflowing corona (e.g., Malzac et al. 2001), or in the RIAF scenario by varying the relative contributions of Bremsstrahlung and inverse Compton (e.g., Quataert et al. 1999).

Similarly, the presence of temporal X-ray variability alone is unable to rule out either scenarios. Indeed, continuous variability on all the sampled timescales is a common characteristic not only of blazars, but also of several classes of radio quiet AGN (most notably, Seyfert and Narrow Line Seyfert 1 galaxies).

On the other hand, if both spectral and temporal information are combined, a markedly different behavior is revealed by radio-quiet and jet-dominated sources. Radio-quiet AGN typically show a softening of the spectrum that accompanies a flux increase (e.g., Papadakis et al. 2002; Markowitz et al. 2003, and references therein), and a more pronounced variability in the soft band than in the hard energy range (Markowitz \& Edelson 2001). Conversely, blazars usually show an opposite spectral trend with a bluer when brighter behavior and with the fractional variability more pronounced at higher energies (e.g., Fossati et al. 2000; Gliozzi et al. 2006; Rebillot et al. 2006).

It must be noticed that intermediate BL Lac objects can sometimes mimic a Seyfert-like spectral variability trend. This occurs when the bandpass of the X-ray detector collects both soft X-rays associated with the highly variable synchrotron component and hard X-rays associated with the slowly variable inverse Compton component (e.g., Tagliaferri et al. 2000; Foschini et al. 2007). However, this unusual (for jet-dominated sources) spectral variability trend is accompanied by an X-ray spectrum parameterized by a concave broken power-law model and hence easily discernible.

\subsection{Flux variability}

We studied the short-term variability using EPIC pn data with time-bins of $500 \mathrm{~s}$ for NGC 1692, PKS 0625-35, and 3C 444. For $3 \mathrm{C} 88$, since the EPIC pn data are severely affected by background flaring, we have used EPIC MOS2 data with time-bins of $1000 \mathrm{~s}$ (given the lower count rate of the MOS data compared to the pn data). The total $0.2-10 \mathrm{keV}$ energy band was divided into a soft $(0.2-1 \mathrm{keV})$ and a hard (1-10 keV) bands, such that the mean count rates in the two sub-bands are comparable and not noise-dominated.

Figure 5 shows the EPIC time series of the $0.2-1 \mathrm{keV}$ (top panels), the $1-10 \mathrm{keV}$ energy band (middle panels), and of the hardness ratio $H R=1-10 \mathrm{keV} / 0.2-1 \mathrm{keV}$ (bottom panels). A visual inspection of Fig. 5 suggests that low-amplitude flux changes are a common phenomenon in the 4 radio galaxies.

In order to quantify the variability in the different energy bands, we have applied $\chi^{2}$ tests and measured the fractional variability $F_{\text {var }}=\left(\sigma^{2}-\Delta^{2}\right)^{1 / 2} /\langle r\rangle$, where $\sigma^{2}$ is the variance, $\langle r\rangle$ the unweighted mean count rate, and $\Delta^{2}$ the mean square value of the uncertainties associated with each individual count rate. Considering the brevity of the observations and the limited count rates, as an operative rule, we deem a time series significantly variable if $P_{\chi^{2}}<0.05$ and marginally variable if $P_{\chi^{2}}=0.1$ (where $P_{\chi^{2}}$ is the probability of constancy associated with a $\chi^{2}$ test).

The results of the variability analysis are reported in Table 6 and can be summarized as follows:

1) In the $0.2-10 \mathrm{keV}$ energy band, only PKS 0625-35, which is the object with the highest count rate and the least contribution from the thermal component, is significantly variable. 3C 88 is marginally variable, whereas no variability is detected in NGC 1692 and 3C 444.

2) In the soft band, both PKS 0625-35 and 3C 88 are significantly variable, whereas NGC 1692 is marginally variable, although the latter result should be considered with caution given the low count rate and the presence of a gap due to background flaring.

3) In the hard band, none of the sources show significant variability, with the exception of $3 \mathrm{C} 88$, which is only marginally variable. The lack of variability in the hard band may be partly related to the lower count rate. On the other hand, PKS 0625-35 (which has an average hard count rate $>1.2 \mathrm{~s}^{-1}$ ) suggests that this difference might be intrinsic. Furthermore, because of the dilution from the thermal component, the variability measured in the soft band should be considered as a lower limit, supporting the hypothesis that the sources are more variable at softer energies.

4) There is some evidence for spectral variability in 3C 88 and $3 \mathrm{C} 444$, suggesting a different temporal behavior in the two energy bands.

\subsection{Spectral variability}

A useful method for investigating the nature of spectral variability is based on the hardness ratio plotted versus the count rate. As an example, Fig. 6 shows the hard/soft X-ray color plotted versus the count rate for NGC 1692, which is the source with the longest EPIC pn coverage. The gray (blue in color) filled circles correspond to time-bins of $500 \mathrm{~s}$. 

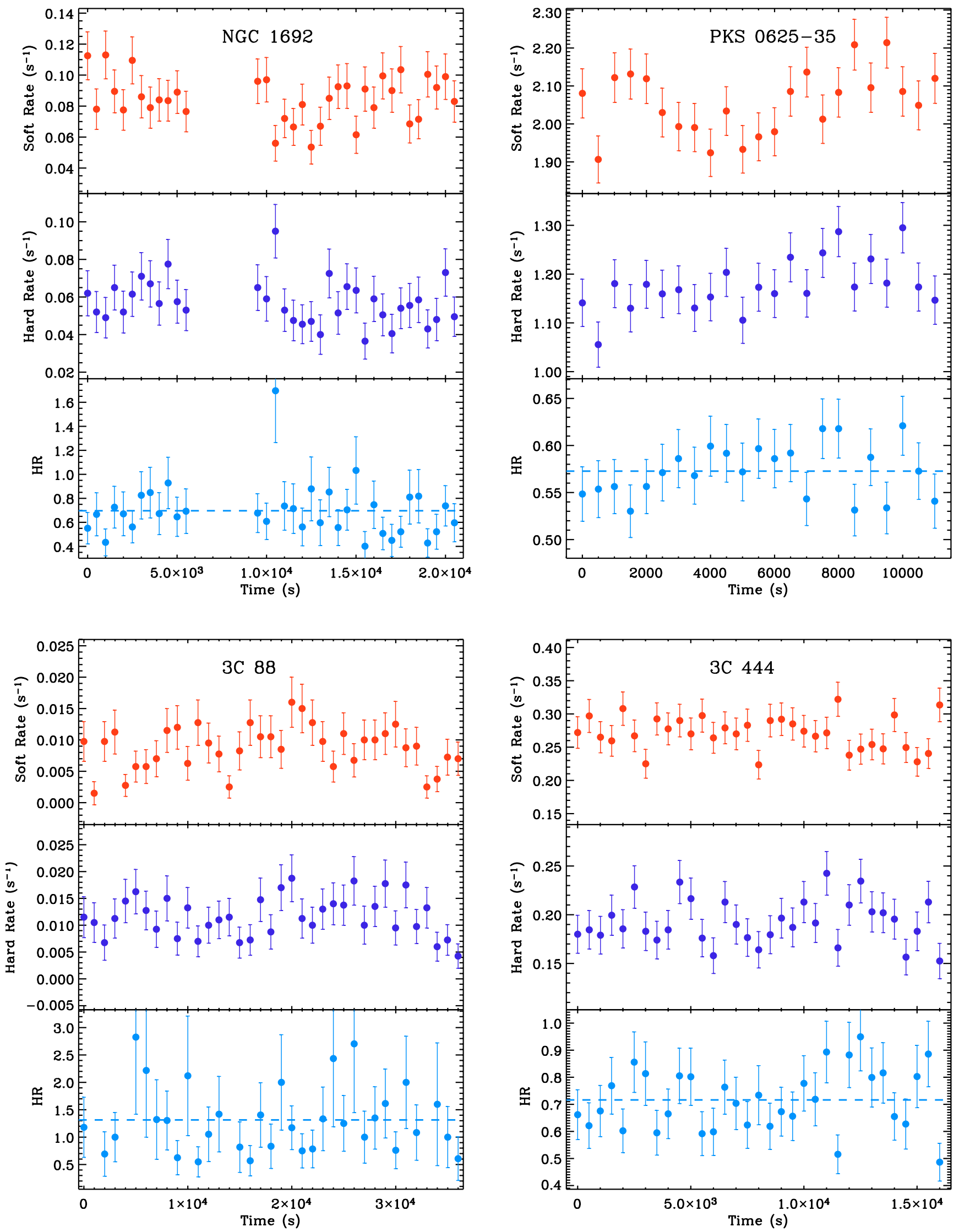

Fig. 5. XMM-NewtonEPIC light curves in the $0.2-1 \mathrm{keV}$ (top panels), $1-10 \mathrm{keV}$ (middle panels) energy bands, and $H R=(1-10 \mathrm{keV} / 0.2-1 \mathrm{keV}$ ) plotted versus the time (bottom panels). For NGC 1692, PKS 0625-35, and 3C 444 we have used EPIC pn data and time bins of 500 s; for 3C 88, EPIC MOS2 data and time bins of 1000 s have been used. 
Table 6. X-ray variability.

\begin{tabular}{lccccccc}
\hline \hline Source & \multicolumn{2}{c}{ Total } & \multicolumn{2}{c}{ Soft } & \multicolumn{2}{c}{ Hard } & HR \\
& $P_{\chi^{2}}$ & $F_{\text {var }}$ & $P_{\chi^{2}}$ & $F_{\text {var }}$ & $P_{\chi^{2}}$ & $F_{\text {var }}$ & $P_{\chi^{2}}$ \\
\hline NGC 1692 & 0.30 & $0.03 \pm 0.07$ & 0.10 & $0.07 \pm 0.05$ & 0.45 & $0.05 \pm 0.10$ & 0.70 \\
PKS 0625-35 & 0.002 & $0.025 \pm 0.006$ & 0.02 & $0.027 \pm 0.009$ & 0.21 & $0.020 \pm 0.015$ & 0.59 \\
3C 88 & 0.10 & $0.10 \pm 0.07$ & $<0.001$ & $0.20 \pm 0.09$ & 0.10 & $0.09 \pm 0.13$ & 0.07 \\
3C 444 & 0.39 & $0.03 \pm 0.03$ & 0.23 & $0.06 \pm 0.03$ & 0.12 & $\ldots$ & 0.02 \\
\hline
\end{tabular}

For NGC 1692, PKS 0625-35 and 3C 444 the variability results have been obtained using EPIC pn light curves with time bins of 500 s. For 3C 88, we have used EPIC MOS2 light curves with time bins of $1000 \mathrm{~s}$, because the EPIC pn data are severely affected by background flaring.

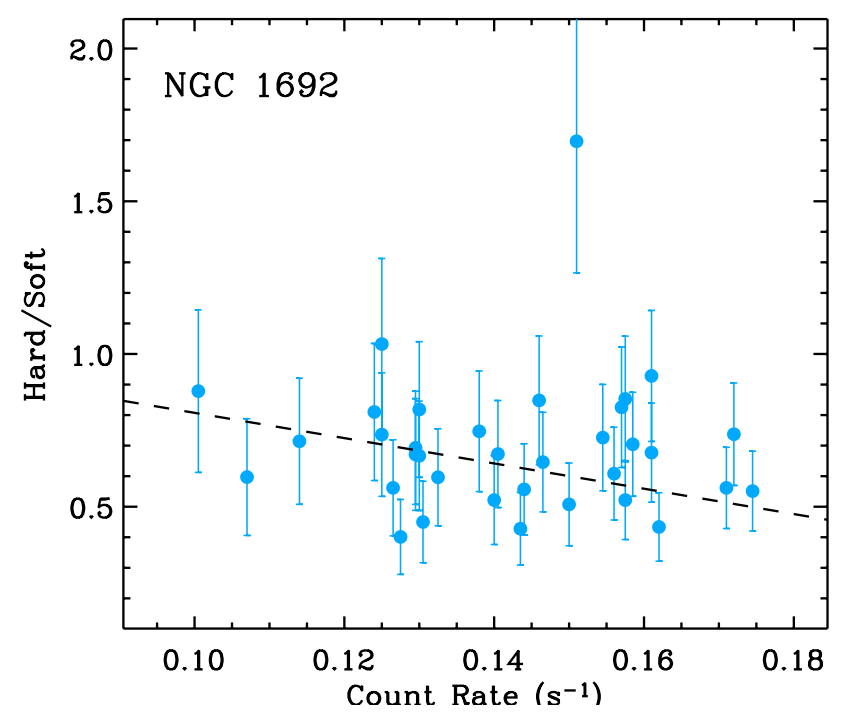

Fig. 6. Hardness ratio (1-10 keV/0.2-1 keV) plotted versus the count rate for NGC 1692. The gray (blue in color) filled circles correspond to time-bins of $500 \mathrm{~s}$. The dashed line represent the best-fit model obtained from a least-squares method.

A visual inspection of Fig. 6 suggests the presence of a weak negative trend with the source softening when the count rate increases. The dashed line represents the best-fit model to the binned data point, obtained from a least-squares method: $H R=(1.2 \pm 0.7)-(4.1 \pm 4.7) r$ (where $r$ is the count rate). As indicated by the large errors, the brevity of the observation and the low amplitude of the flux variations hamper this kind of analysis, and no firm conclusions can be derived on the presence of a trend in the $H R-c t$ plot. Nevertheless, compared to a constant model (i.e., a model with slope $=0$ and intercept $=\langle H R\rangle$ ), the best-fit linear model improves the data fit by $\Delta \chi^{2} \sim 4$ for one additional degree of freedom.

Since AGN are generally characterized by red-noise variability (i.e., the variability is more pronounced on longer timescales), in order to successfully perform this kind of analysis, each source would require two observations performed by the same instrument months/years a part, in order to increase the chances of observing substantial flux and spectral changes.

For technical reasons the exposure of PKS 0625-35 was split into two observations (of duration of $\sim 1.5 \mathrm{ks}$ and $\sim 13 \mathrm{ks}$, respectively) taken at beginning of May 2005 and toward the end of August 2005. Although the shortness of the first observation does not allow to properly constrain the spectral parameters (due to their large uncertainties, they are consistent with those obtained during the second observation), it yields enough counts to investigate the spectral variability using the hardness ratio. However, since during the first observation, the EPIC pn camera did not provide valid data, the comparison between the hardness ratio has been performed using MOS data only. For May 2005, when the average EPIC MOS count rate was $r_{\mathrm{t} 1}=0.74 \pm 0.02 \mathrm{~s}^{-1}$, the hardness ratio was $H R_{\mathrm{t} 1}=1.10 \pm 0.04$, whereas 4 months later the increase of the average count rate by $\sim 10 \%, r_{\mathrm{t} 2}=0.81 \pm 0.01 \mathrm{~s}^{-1}$, was accompanied by an the hardness ratio $H R_{\mathrm{t} 2}=0.97 \pm 0.01$, yielding an apparently significant decrease of $H R_{\mathrm{t} 1}-H R_{\mathrm{t} 2}=0.13 \pm 0.04$. In other words, the two XMM-Newton observations suggest that PKS 0625-35 follows the typical Seyfert-like, redder when brighter behavior.

\section{Broad-band properties}

In addition to the combination of the X-ray spectral and temporal results, important insights into the AGN central engine can be obtained by analyzing the broadband properties, such as SEDs, broadband spectral indices, and radio loudness parameters.

Indeed, the modeling of SEDs ranging from the radio to the $\gamma$-ray energies has proved to be one of the most effective tools to investigate the physics of blazars (e.g., Ghisellini et al. 2002). The study of SEDs has also been vastly applied to investigate the nature of the accretion flow in AGN and specifically to discriminate between radiatively efficient and inefficient scenarios (e.g., Quataert et al. 1999). Finally, the study of broadband SEDs has been frequently applied to the scaled-down version of the AGN, the Galactic black hole (GBHs), to investigate the jet contribution at X-rays in the low/hard spectral state (e.g., Markoff et al. 2003). However, despite the much higher signal-to-noise of the GBH data, the X-ray jet dominance is still matter of strong debate (Zdziarski et al. 2003). The fact that the discussion is still open for GBHs, the objects with the highest $\mathrm{S} / \mathrm{N}$ and broadest energy coverage, along with the spectral degeneracy already discussed in Sect. 4, indicates that not even SED studies can be considered exhaustive diagnostics when used in isolation.

In general, two additional problems plague the investigation of AGN SEDs: 1) the relative contributions from different energy bands (and hence the overall SED) are known to evolve in time, casting doubts on the results obtained from non-simultaneous SED studies; and 2) the poor (or lack of) coverage of the UV band, which is thought to map the emission from the accretion disk and thus of crucial importance for AGN studies (Malkan 1983).

XMM-Newton, with the EPIC cameras and the OM operating at the same time, offers the ideal solution to these problems since it covers simultaneously the $0.2-10 \mathrm{keV} X$-ray range and the optical/UV band. 
Table 7. Optical and UV magnitudes.

\begin{tabular}{|c|c|c|c|c|}
\hline Source & Filter $(\lambda)$ & $\begin{array}{l}\text { Observed } \\
\text { magnitude }\end{array}$ & $\begin{array}{c}\text { De-reddened } \\
\text { magnitude }(\mathrm{SMC})^{a}\end{array}$ & $\begin{array}{c}\text { De-reddened } \\
\text { magnitude }(\mathrm{X} \text {-ray })^{b}\end{array}$ \\
\hline \multirow[t]{3}{*}{ NGC 1692} & $U(3440 \AA)$ & 16.4 & 16.2 & 13.1 \\
\hline & UVW1 (2910 Å) & 19.4 & 19.1 & 16.0 \\
\hline & UVM2 (2310 Å) & $>22.0$ & $>21.6$ & $>18.5$ \\
\hline \multirow[t]{3}{*}{ PKS 0625-35 } & $U(3440 \AA)$ & 15.9 & 15.6 & 15.0 \\
\hline & UVW1 (2910 Å) & 15.7 & 15.3 & 14.7 \\
\hline & UVM2 (2310 Å) & 15.8 & 15.2 & 14.6 \\
\hline \multirow[t]{3}{*}{$3 \mathrm{C} 88^{c}$} & $U(3440 \AA)$ & 18.6 & 18.0 & 18.1 \\
\hline & UVW1 (2910 Å) & 18.6 & 17.8 & 18.0 \\
\hline & UVM2 (2310 Å) & 18.6 & 17.4 & 17.6 \\
\hline \multirow[t]{3}{*}{$3 \mathrm{C} 444$} & $U(3440 \AA ̊)$ & 19.4 & 19.3 & 18.7 \\
\hline & UVW1 (2910 Å) & 19.2 & 19.0 & 18.5 \\
\hline & UVM2 (2310 Å) & 19.6 & 19.4 & 18.8 \\
\hline
\end{tabular}

${ }^{a}$ Magnitude de-reddened using a SMC-type extinction law (see text for details). ${ }^{b}$ Magnitude de-reddened using a reddening $A(V) / N_{\mathrm{H}}=5.3 \times 10^{-22}$ with $N_{\mathrm{H}}$ derived fromthe X-ray spectral fitting. ${ }^{c}$ For $3 \mathrm{C} 88$ only the $U$ filter yielded a value for the magnitude. The same magnitude was used for the other 2 filters to allow a direct comparison with the other targets.

\subsection{SEDs}

The intrinsic shape of the optical-UV continuum in AGN is still a matter of debate, because there is no general consensus on the extinction corrections that need to be applied. For completeness, we have tried different extinction curves, including the Galactic extinction proposed by Cardelli et al. (1989), the reddening proposed by Gaskell et al. (2004), and the Small Magellanic Cloud (SMC) type extinction (Prévot et al. 1984). The results from the different extinction laws are consistent with each other within 10-20\%. In the following, we will make use of the de-reddened values obtained with a SMC-type extinction, since it proved to work better than the other extinction curves on a large sample of AGN from the Sloan Digital Sky Survey (Hopkins et al. 2004). We also applied extinction corrections by assuming the absorption column density derived from the X-ray spectral fits, $N_{\mathrm{H}}$, and converting it into optical extinction with the relation $A(V) / N_{\mathrm{H}}=5.3 \times 10^{-22}$ (Cox \& Allen 2000).

The observed magnitudes and the de-reddened values obtained using the SMC-type extinction and $N_{\mathrm{H}}$ from the X-ray analysis are summarized in Table 7 . For $3 \mathrm{C} 88$ the values should be considered only as suggestive, since only the $U$ filter yielded a value for the magnitude. The same value was used for the other 2 filters only to allow a direct comparison with the other targets in the 3 optical/UV bands. In addition, unlike the other sources, the $U$ image of 3C 88 shows a diffuse and uniform emission without any clear enhancement in the central region. This may indicate either that the nuclear optical/UV emission is completely absorbed by the high column density suggested by the $N_{\mathrm{H}}-\Gamma$ contour plots obtained from the X-ray spectroscopy, or that the nuclear emission is intrinsically too faint to be detectable.

Figure 7 shows the SEDs of the 4 WLRGs from the radio to the X-rays. Optical/UV and X-ray data are simultaneous, whereas the radio data are taken from the literature. The two sets of the optical/UV data for NGC 1692, PKS 0625-35, and 3C 444 correspond to the SMC-type and X-ray extinction corrections, respectively.
The nuclear emission of weak radio galaxies is commonly ascribed to a combination of an ADAF (or more generally a RIAF) component and the non-thermal continuum expected from the base of the relativistic jet, with the two emissions possibly dominating in different energy bands (e.g. Quataert et al. 1999). On the other hand, it is also conceivable, as discussed in the Introduction, that optical emission from LINERS is dominated by a standard accretion flow.

The SEDs of the four sources analyzed in this work share some similarities (the optical seems to dominate the bolometric output) but are also different, especially in the X-ray band. A detailed modeling of the overall emission is beyond the scope of this paper (for a recent work see e.g. Wu et al. 2007). As an example, we report a possible modeling of the emission from PKS 0625-35, whose overall shape is rather suggestive of a dominance of the non-thermal synchrotron continuum of the jet from the radio to the X-ray band. Evidence for the presence of a bright jet also comes from VLBA observations (e.g. Venturi et al. 2000).

The model assumes a spherical emission region (with radius $R$ ), in motion with bulk Lorentz factor $\Gamma$ at an angle $\theta$ with respect to the line of sight (the latter parameters are combined in the Doppler relativistic factor $\delta$ ). The source is filled by tangled magnetic field with intensity $B$ and by relativistic electrons assumed to follow a smoothed broken power-law energy distribution with normalization $K$, extending from $\gamma_{\min }$ to $\gamma_{\max }$ and with indices $n_{1}$ and $n_{2}$ below and above the break located at $\gamma_{\mathrm{b}}$. This model is clearly rather simplified. As discussed by Ghisellini et al. (2005) we expect that subpc scale FRI jets have a complex structure: The emission of radio-galaxies, in which the jet is misaligned, is possibly dominated by the lateral walls (the "layer") of the jet, surrounding the faster and more beamed spine responsible for the emission of blazars, the aligned version of radiogalaxies.

The curve reported in Fig. 7 assumes the following values: $R=6 \times 10^{16} \mathrm{~cm}, \delta=4, B=0.3 \mathrm{G}, K=3 \times 10^{2} \mathrm{~cm}^{-3}, \gamma_{\min }=1$, $\gamma_{b}=3 \times 10^{4}, \gamma_{\max }=2 \times 10^{6}, n_{1}=2, n_{2}=4$. The power 

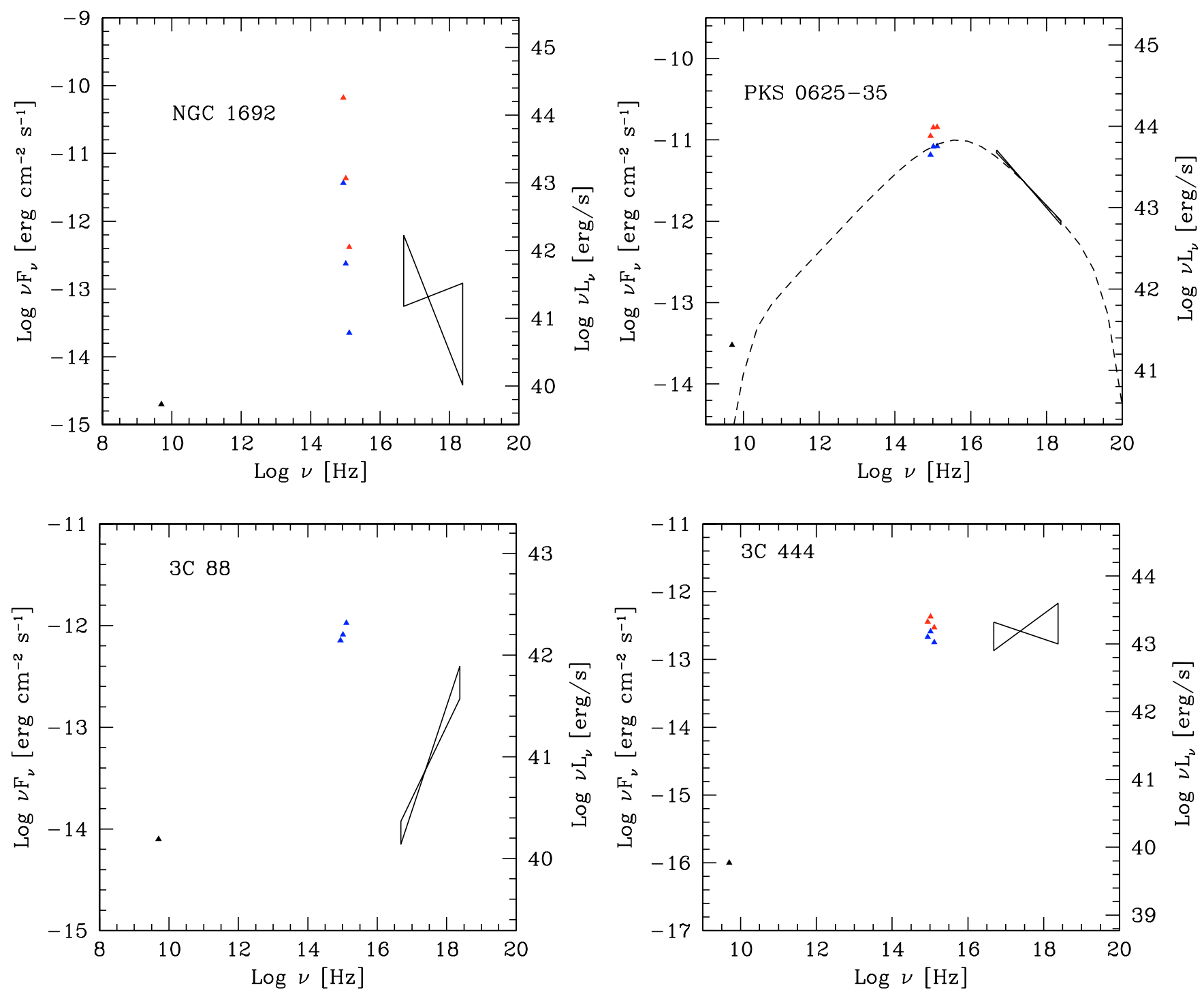

Fig. 7. Spectral energy distributions of the 4 radio galaxies/LINERs. The optical/UV data are from the OM aboard XMM-Newton and thus simultaneous to the X-ray data. The radio data are taken from the literature. The two sets of the optical/UV data (red and blue in colors) for NGC 1692, PKS 0625-35, and 3C 444 correspond to two different extinction corrections (see text for more details). The dashed line superimposed to the PKS 0625-35 data represents the best fit from the jet model described in the text.

carried by the jet, calculated assuming the presence of 1 proton per emitting electron (e.g. Maraschi \& Tavecchio 2003) is $P_{\mathrm{j}}=$ $2.5 \times 10^{45} \mathrm{erg} / \mathrm{s}$. Note that the curve underestimates the radio flux since in this band the emission is self-absorbed. As generally assumed in the case of sub-pc scale jets, the emission in the radio band is probably the sum of the emission from larger portions of the jet.

\subsection{UV $-X$-ray constraints}

Recently, Steffen et al. (2006) showed for a sample of optically selected (Seyfert-like) AGN that the broadband spectral index $\alpha_{\mathrm{OX}}$ is strongly correlated with the UV monochromatic luminosity, suggesting the existence of AGN spectral evolution with the luminosity. Maoz (2007) performed a similar study for a sample of unobscured LINERs showing that they follow a similar trend. Here, we apply the same approach to our sample by computing the spectral index, $\alpha_{\mathrm{OX}}=$ $\log \left(l_{2500 \AA} / l_{2 \mathrm{keV}}\right) / \log \left(v_{2500} \AA / v_{2} \mathrm{keV}\right)$ (Tananbaum et al. 1979), and plotting it versus the UV monochromatic luminosity. The values of the flux at $2500 \AA$ have been obtained by converting the flux measured in the UVM2 band $(2310 \AA)$ assuming a typical slope of $0.7\left(f_{v} \propto v^{-0.7}\right)$.

The values of $\alpha_{\text {OX }}$, computed assuming the two extinction prescriptions discussed above, are summarized in Table 8 and plotted in Fig. 8, superimposed to the best-fit linear regression found by Steffen et al. (2006). 3C 444, PKS 0625-35, and NGC 1692 show a broad agreement with the best-fit linear correlation suggesting a similarity (or at least a smooth transition) with Seyfert-like objects. On the other hand, no firm conclusions can be drawn for the remaining $3 \mathrm{C} 88$, due to the large uncertainty of its UV luminosity.

\subsection{Radio constraints}

Important constraints on the nature of radio galaxies/LINERs can be obtained by combining our XMM-Newton data with archival radio information, keeping in mind that the data are not simultaneous. 
Table 8. Broadband properties.

\begin{tabular}{lccccc}
\hline \hline \multirow{2}{*}{ Source } & \multicolumn{2}{c}{$\alpha_{\mathrm{OX}}$} & \multicolumn{2}{c}{$\log R_{\mathrm{UV}}^{a}$} & $\log R_{\mathrm{X}}^{b}$ \\
& $\mathrm{SMC}^{c}$ & $\mathrm{X}^{- \text {ray }^{d}}$ & $\mathrm{SMC}^{c}$ & $\mathrm{X}_{\text {-ray }}{ }^{d}$ & \\
\hline NGC 1692 & -0.92 & -1.39 & 4.33 & 3.07 & -1.27 \\
PKS 0625-35 & -1.17 & -1.26 & 2.94 & 2.71 & -1.98 \\
3C 88 & -1.38 & $\ldots$ & 3.26 & $\ldots$ & -1.50 \\
3C 444 & -0.85 & -0.94 & 2.60 & 2.38 & -3.77 \\
\hline
\end{tabular}

${ }^{a} \mathrm{UV}$ radio loudness $R_{\mathrm{UV}}=L_{v}(5 \mathrm{GHz}) / L_{v}(2500 \AA) .{ }^{b} \mathrm{X}$-ray radio loudness $R_{\mathrm{X}}=v L_{v}(5 \mathrm{GHz}) / L_{2-10 \mathrm{keV} .}{ }^{c}$ Computed assuming a SMC-type extinction curve. ${ }^{d}$ Computed assuming $A(V) / N_{\mathrm{H}}=5.3 \times 10^{-22}$ with $N_{\mathrm{H}}$ derived from the X-ray spectral fitting.

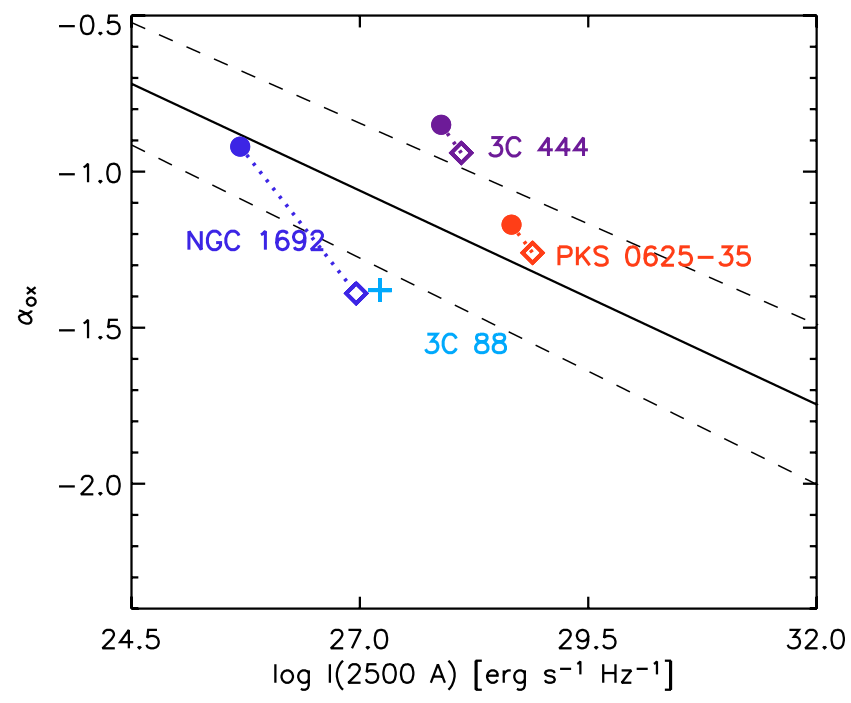

Fig. 8. $\alpha_{\text {OX }}$ plotted versus $2500 \AA ̊$ monochromatic luminosity. The black continuous line corresponds to the best-fit linear regression found by Steffen et al. (2006) and the dashed lines account for the uncertainties in the fit. Filled circles correspond to values obtained assuming Galactic extinction correction, whereas open diamonds correspond to values obtained assuming the extinction provided by the X-ray spectral fitting. For completeness, also 3C 88 has been shown, however its $\alpha_{\mathrm{OX}}$ value should be regarded as highly uncertain (see text for details).

For example, a possible way to explore the origin of the $\mathrm{X}$-ray luminosity (associated with the power-law component) in radio galaxies is to plot it versus the radio core dominance, $\mathfrak{R}=L_{\text {core }} / L_{\text {lobe }}$, which is commonly used as indicator of the jet inclination (the larger $\mathfrak{R}$ the smaller the inclination angle). According to the classification proposed by Baker \& Hunstead (1995) based on $\mathfrak{R}$, radio-loud objects can be divided into 3 subsets: face-on objects (likely jet-dominated) with $\mathfrak{R} \geq 1$, intermediate objects $(0.1<\mathfrak{R}<1)$, and edge-on objects $(\mathfrak{R} \leq 0.1)$. All our sources have relatively low values, as expected for radio galaxies. Specifically, NGC 1692, 3C 88, and 3C 444 fall in the edge-on category, whereas PKS $0625-35$ is classified as an intermediate object (see last column of Table 1).

This approach has been used by Kharb \& Shastri (2004) to investigate the origin of the optical nuclear emission in a sample of radio galaxies observed with $H S T$. The strong positive correlation prompted these authors to conclude that the optical nuclear emission is strongly beamed and likely to be related to the unresolved base of the jet. On the other hand, the same analysis carried out by Rinn et al. (2005) for the X-ray luminosity of a

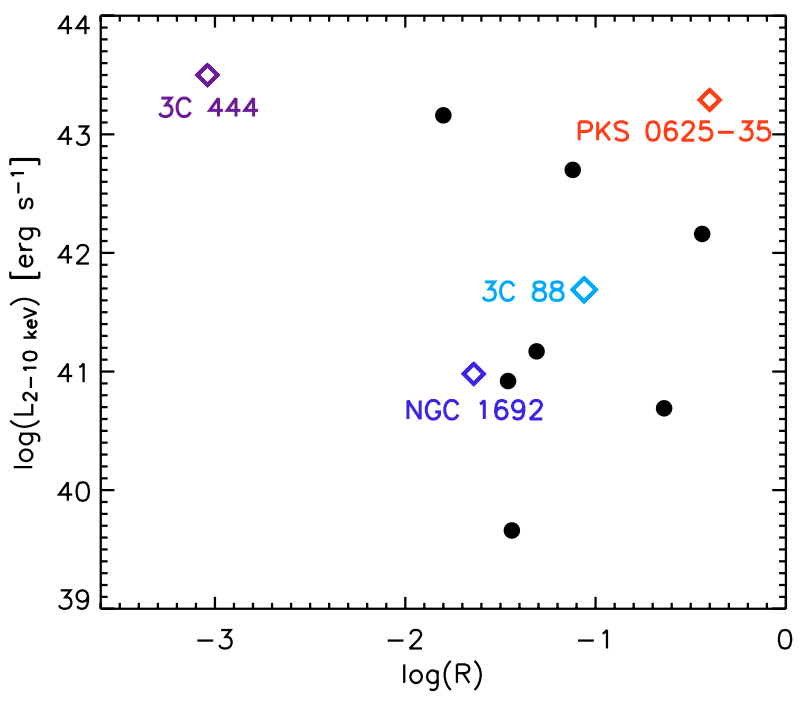

Fig. 9. 2-10 X-ray luminosity associated with the power-law component plotted against the radio core dominance. Open diamonds are the 4 sources analyzed in this work. Filled circles refers to weak-line radio galaxies/LINERs analyzed by Rinn et al. (2005).

sample of radio galaxies/LINERs did not revealed any positive trend between $L_{2-10 \mathrm{keV}}$ and $R$, suggesting that the X-ray emission is not beamed and hence more likely to be related to the accretion flow.

Given the common classification of the sources belonging to the sample analyzed by Rinn et al. (2005) and ours (all are radio galaxies whose nuclei are optically classified as LINERs), we have combined the 2 samples considering only the most reliable X-ray and radio data. The results, plotted in Fig. 9, clearly indicate the lack of any correlation. This is confirmed by a Spearman rank correlation analysis that yields $\tau=-0.15$ with a chance probability of $\sim 68 \%$.

Further useful information can be provided by the radio loudness parameter. Originally, the radio loudness, $R_{\mathrm{O}}$, was defined by the ratio of the $5 \mathrm{GHz}$ to the optical $B$ band flux densities and used to discriminate between radio-quiet and radio-loud $\left(R_{\mathrm{O}}>10\right.$; Kellermann et al. 1989). More recently, other definitions of radio loudness have been introduced, making use of energy bands less affected by absorption $\left(R_{\mathrm{X}}=v L_{v}(5 \mathrm{GHz}) / L_{2-10 \mathrm{keV}}\right.$; Terashima \& Wilson 2003) or with lower galaxy contribution $\left(R_{\mathrm{UV}}=L_{v}(5 \mathrm{GHz}) / L_{v}(2500 \AA)\right.$; Maoz 2007) than the $B$ band.

In his analysis of the SEDs of 7 low-power AGN/LINERs, Ho (1999) pointed out the relatively high radio loudness of these 
objects, suggesting the existence of an inverse relationship between radio loudness and Eddington ratio. This relationship has been confirmed by several observations, which have also revealed the existence of two parallel trends in the $R_{\mathrm{O}}$-Eddington ratio plot: An upper "radio-loud" branch populated by FRI, FRII, and radio-loud QSO, and a lower "radio-quiet" branch with LINERs, Seyfert galaxies and radio-quiet quasars (see Sikora et al. 2007, and references therein).

Recently, Panessa et al. (2007) investigated the radio properties of a large sample of Seyfert galaxies and LINERs using both $R_{\mathrm{O}}$ and $R_{\mathrm{X}}$. They confirmed the existence of a strong anti-correlation between radio loudness and Eddington ratio and empirically determined the threshold between radio-quiet and radio-loud objects in the $\log R_{\mathrm{X}}-\log L_{2-10 \mathrm{keV}}$ plot to be around $\log R_{\mathrm{X}} \sim-2.75$. Similarly, Maoz (2007), extending the Sikora et al. (2007) plane to the UV band, showed that the unobscured LINERs observed with $H S T$ preferentially lie on the radio-quiet branch of the $\log R_{\mathrm{UV}}-\log L_{\mathrm{UV}}$ plot.

We computed $R_{\mathrm{X}}$ and $R_{\mathrm{UV}}$ for our sample (the results are summarized in Table 8). Our results confirm the anti-correlation between radio loudness and Eddington ratio. As for the location of our targets in the $\log R_{\mathrm{UV}}-\log L_{\mathrm{Uv}}$ plot, we found that $3 \mathrm{C} 444$ lies well within the radio-quiet region/branch, PKS 0625-35 is in between the 2 branches, whereas NGC 1692 and 3C 88 are more consistent with the bottom part of the radio-loud branch. However, once more the UV values for the latter 2 objects are severely affected by the large uncertainty of the extinction.

\section{Summary and conclusions}

We have investigated the nature of 4 WLRGs, NGC 1692, PKS 0625-35, 3C 88, 3C 444, using observations from XMMNewton, and combining information from the time-averaged spectral analysis, from the temporal and spectral variability, and from broadband studies. The main results can be summarized as follows:

- The 4 AGN have Eddington ratios $L_{\text {bol }} / L_{\text {Edd }}$ that span 2 orders of magnitude ranging between $\sim 1 \times 10^{-5}$ (NGC 1692) and $2-3 \times 10^{-3}$ (PKS 0625-35 and 3C 444), with 3C 88 having an intermediate value of $\sim 2 \times 10^{-4}$.

- The 4 targets are adequately fitted by the same continuum model that comprises at least one thermal component $(k T \sim 0.65-1.45 \mathrm{keV})$ and a partially absorbed power law. However, the relative contributions of the individual spectral components and the photon indices change significantly from source to source. For example, NGC 1692 and PKS 0625-35 have fairly steep power-law components ( $\Gamma \sim 2.5-2.9$ ), whereas $3 \mathrm{C} 88$ has a flat photon index $(\Gamma \sim 1.1)$, and $3 \mathrm{C} 444$ is the only one showing a typical Seyfert-like $\Gamma \sim 1.9$ accompanied by an apparent line-like excess around $6.7 \mathrm{keV}$. If this excess is fitted with a Gaussian model, the resulting line is centered around $6.7 \mathrm{keV}$, fairly strong $(E W \sim 550 \mathrm{eV})$ and possibly broad $(\sigma \sim 0.35 \mathrm{keV})$. However, observations with a better coverage of the hard $\mathrm{X}$-ray band and with longer duration are necessary to firmly establish the presence of a broad line and constrain the line parameters

- There is evidence only for moderate absorption $\left(N_{\mathrm{H}} \sim\right.$ $10^{21} \mathrm{~cm}^{-2}$ ) in NGC 1692, PKS 0625-35, and 3C 444. On the other hand, the flat photon index derived for $3 \mathrm{C} 88$ and the $N_{\mathrm{H}}-\Gamma$ contour plots suggest that this source might have a larger column density of the order of $N_{\mathrm{H}} \sim 10^{22} \mathrm{~cm}^{-2}$.
- The results of the variability analysis indicate that PKS $0625-35$ is significantly variable in the $0.2-10 \mathrm{keV}$ and $0.2-1 \mathrm{keV}$ energy bands, whereas $3 \mathrm{C} 88$ is variable in the soft band only, and NGC 1692 is marginally variable in the soft band. In general, there is a suggestive evidence that the sources are more variable in the soft band, although the limited statistics due to the short observations does not allow to draw firm conclusions.

- For PKS 0625-35, the split of the exposure into two observations taken 4 months a part, makes it possible to investigate the spectral variability using the hardness ratio. The spectrum becomes softer as the source brightens, following the typical Seyfert-like spectral behavior.

- The broadband SED of PKS 0625-35, comprising (nonsimultaneous) radio data combined with (simultaneous) UV and X-ray measurements, can be adequately fitted with a single synchrotron component with little contribution from the putative accretion disk. However, the paucity of data-points does not allow one to rule out alternative scenarios.

- For 3C 444, PKS 0625-35, and NGC 1692, the values of $\alpha_{\mathrm{OX}}$ plotted versus the UV monochromatic luminosity follow the best-fit linear regression found by Steffen et al. (2006) for Seyfert galaxies. This suggests a similarity (or at least a smooth transition) with Seyfert-like objects. No firm conclusions can be drawn for the remaining object $3 \mathrm{C} 88$, due to the large uncertainty of its UV luminosity.

- The lack of a positive correlation between $L_{2-10 \mathrm{keV}}$ and the viewing angle, parameterized by the radio core dominance, suggests that the X-ray emission is not beamed and hence more likely to be related to the accretion flow.

- The study of the X-ray and UV radio loudness indicates that 3C 444 lies well within the radio-quiet region/branch, PKS $0625-35$ is in between the 2 branches, whereas NGC 1692 and 3C 88 are more consistent with the radioloud branch.

Before concluding, we can try to combine the different findings from our analysis in an attempt of answering the fundamental question on the origin of the X-ray emission in WLRGs.

Several pieces of evidence seem to favor an accretion-related origin. First, relatively high values of $L_{X} / L_{E d d}$, as those found for 3 of our targets, are commonly interpreted as Comptonized emission from a RIAF, based on studies of LLAGN and GBHs in the low/hard state (Yuan \& Cui 2005; Wu et al. 2007). Second, the lack of a positive correlation in the $L_{X}-R$ plane (see Fig. 9), suggests that the $\mathrm{X}$-ray emission associated with the power law component is not beamed and hence unlikely to be produced by a relativistic jet. Third, the results from the temporal and spectral variability analyses (more pronounced variability in the soft band and the spectral softening associated with the source brightening observed in PKS 0625-35) are more consistent with the trends observed in Seyfert galaxies and at odds with the typical blazar-like behavior. Finally, the time-averaged spectral results of $3 \mathrm{C} 444(\Gamma \sim 1.9$ and possibly broad $\mathrm{Fe} \mathrm{K} \alpha$ ), coupled with its radio-quiet nature and the relatively high luminosity and Eddington ratio, suggest that not only this source is unlikely jetdominated but also that the accretion mode might be similar to the Seyfert-like one (i.e., radiatively efficient).

On the other hand, a significant (or even dominant) contribution from the jet in the X-ray regime cannot be a priori excluded at least for some objects. For example, the main properties of NGC $1692-L_{\mathrm{X}} / L_{\mathrm{Edd}} \sim 8 \times 10^{-7}, \Gamma \sim 2.9$ and strong radio-loudness - seem to naturally fit the jet-dominated scenario. Similarly, the SED and the steep $\Gamma$ of PKS 0625-35, coupled 
with the relatively small viewing angle, might be adequately interpreted in the framework of jet-dominated models.

In summary, based on the analysis of time-averaged spectra combined with model-independent information from X-ray temporal and spectral variability, and with inter-band information, we cannot derive a general conclusion valid for the entire sample. In fact, the main conclusion of this work is that LINERs represent a very heterogeneous class. Not only they comprise non-AGN and AGN objects, not only the AGN class can be divided into radio-quiet and radio-loud LINERs, but also the latter subclass seems to encompass quite different objects. These may range from intrinsically low-luminosity objects (in terms of Eddington ratio), to objects potentially strongly absorbed, to brighter objects with properties more in line with Seyfert galaxies. Our results are based on a very small sample, which needs to be extended to derive more general conclusions. Nevertheless, it demonstrates the need of high-quality X-ray observations to disentangle the relative contribution of the different components in order to shed some light on the nature of low-power radio galaxies.

Acknowledgements. We thank the referee for the comments and suggestions that improved the clarity of the paper. We are grateful to Ari Laor, Ramesh Narayan, and Matthew Malkan for interesting discussions and useful suggestions. M.G. acknowledges support by the XMM-Newton Guest Investigator Program under NASA grants 200866 and 201101. Funds from the NASA LTSA grant NAG510708 are also gratefully acknowledged.

\section{References}

Arnaud, K. 1996, in Astronomical Data Analysis Software and Systems V, ed. G. Jacoby \& J. Barnes (San Francisco: ASP), ASP Conf. Ser., 101, 17

Baker, J. C., \& Hunstead, R. W. 1995, ApJ, 452, L95

Balmaverde, B., Capetti, A.; \& Grandi, P., et al. 2006, A\&A, 451, 35

Bettoni, D., Falomo, R., Fasano, G., \& Govoni, F. 2003, A\&A, 399, 869

Bennet, C. L., Halpern, M., Hinshaw, G., et al. 2003, ApJS, 148, 1

Cardelli, J. A., Clayton, G. C., \& Mathis, J. S. 1989, ApJ, 345, 245

Chiaberge, M., Capetti, A, \& Celotti, A. 2002, A\&A, 394, 791

Cox, A. N., \& Allen, C. W. 2000, Allen's Astrophysical Quantities, IV edition (New York: AIP)

Di Matteo, T., Johnstone, R. M., Allen, S. W., \& Fabian, A. C. 2001, ApJ, 550, L19

Di Matteo, T., Allen, S. W., Fabian, A. C., Wilson, A. S., \& Young, A. J. 2003, ApJ, 582, 133

Donato, D., Sambruna, R. M., \& Gliozzi, M. 2004, ApJ, 617, 915

Dopita, M. A., \& Sutherland, R. S. 1995, ApJ, 455, 468

Dorman, B., \& Arnaud, K. A. 2001, in Astronomical Data Analysis Software and Systems X, ed. F. R. Harnden, Jr., F. A. Primini, \& H. E. Payne (San Francisco: ASP), ASP Conf. Proc., 238, 415

Dudik, R. P., Satyapal, S., Gliozzi, M., \& Sambruna, R. M. 2005, ApJ, 620, 113

Elvis, M., Wilkes, B. J., McDowell, J. C., et al. 1994, ApJS, 95, 1

Eracleous, M., \& Halpern, J. P. 2001, ApJ, 554, 240

Evans, D. A., Worrall, D. M., Hardcastle, M. J., Kraft, R. P., \& Birkinshaw, M. 2006, ApJ, 642, 96

Foschini, L., Tagliaferri, G., Pian, E., et al. 2006, A\&A, 455, 871

Fossati, G., Celotti, A., Chiaberge, M., et al. 2000, ApJ, 541, 153

Filippenko, A. V., \& Halpern, J. P. 1984, ApJ, 285, 458
Gaskell, C. M., Goosmann, R., Antonucci, R. R. J., \& Whysong, D. H. 2004, ApJ, 616, 147

Gebhardt, K., Richstone, D., Tremaine, S., et al. 2003, ApJ, 583, 92

Ghisellini, G., Celotti, A., \& Costamante, L. 2002, A\&A, 386, 833

Ghisellini, G., Tavecchio, F., \& Chiaberge, M. 2005, A\&A, 432, 401

Gliozzi, M., Sambruna, R. M., \& Brandt, W. N. 2003, A\&A, 408, 949

Gliozzi M., Sambruna, R. M., Jung, I., et al. 2006, ApJ, 646, 61

Guainazzi, M., Bianchi, S., \& Dovčiak, M. 2006, AN, 327, 1032

Haardt, F., Maraschi, L., \& Ghisellini, G. 1994, ApJ, 432, L95

Hardcastle, M. J., Evans, D. A., \& Croston J. H. 2006, MNRAS, 370, 1893

Heckman, T. M. 1980, A\&A, 87, 152

Ho, L. C. 1999, ApJ, 516, 672

Ho, L. C., Filippenko, A. V., \& Sargent, W. L. W. 1997, ApJ, 487, 568

Hopkins, P. F., Strauss, M. A., Hall, P. B., et al. 2004, AJ, 128, 1112

Jackson, N., \& Rawlings S. 1997, MNRAS, 286, 241

Kellermann, K. I., Sramek, R., Schmidt, M., Shaffer, D. B., \& Green, R. 1989, ApJ, 98, 4

Kirsch, M. 2006, EPIC status of calibration and data analysis, XMM-SOC-CALTN-0018, v. 2.5

Kharb, P., \& Shastri, P. 2004, A\&A, 425, 825

Lewis, K., Eracleous, M., \& Sambruna, R. M. 2003, ApJ, 593, 115

Magorrian, J., Tremaine, S., Richstone, D., et al. 1998, AJ, 115, 2285

Malkan, M. A. 1983, ApJ, 268, 582

Malzac, J., Beloborodov, A. M., \& Poutanen, J. 2001, MNRAS, 326, 417

Maoz, D. 2007, MNRAS, 377, 1696

Maraschi, L., \& Tavecchio, F. 2003, ApJ, 593, 667

Marconi, A., Risaliti, G., Gilli, R., et al. 2004, MNRAS,351, 169

Markoff, S., Nowak, M., Corbel, S., Fender, R., \& Falcke, H. 2003, New Astron. Rev., 47, 491

Markowitz, A., \& Edelson, R. 2001, ApJ, 547, 684

Markowitz, A., Edelson, R., \& Vaughan, S. 2003, ApJ, 598, 935

Mason, K. O., Breeveld, A., Much, R., et al. 2001, A\&A, 365, L36

Nagar, N. M., Falcke, H., \& Wilson, A. S. 2005, A\&A, 435, 521

Papadakis, I. E., Petrucci, P. O., Maraschi, L., et al. 2002, ApJ, 573, 92

Pellegrini, S. 2005, ApJ, 624, 155

Prévot, M. L., Lequex, J., Maurice, E., Prévot, L., \& Rocca-Volmerange B. 1984, A\&A, 132, 389

Quataert, E., Di Matteo, T., \& Narayan, R. 1999, ApJ, 525, L89

Rebillot, P. F., Badran, H. M., Blaylock, G., et al. 2006, ApJ, 641, 740

Rinn, A., Sambruna, R. M., \& Gliozzi, M. 2005, ApJ, 621, 167

Satyapal, S., Sambruna, R. M.; \& Dudik, R. P. 2004, A\&A, 414, 825

Sikora, M., Stawarz, L., \& Lasota, J. 2007, ApJ, 658, 815

Smith, R. K., Brickhouse, N. S., Liedahl, D. A., \& Raymond, J. C. 2001, ApJ, 556, L91

Steffen, A. T., Strateva, I., Brandt, W. N., et al. 2006, AJ, 131, 2826

Strüder, L., Briel, U., Dennerl, K., et al. 2001, A\&A, 365, L18

Tadhunter, C. N., Morganti, R., Robinson, A., Dickson, R., et al. 1998, MNRAS, 298, 1035

Tagliaferri, G., Ghisellini, G., Giommi, P., et al. 2000, A\&A, 354, 431

Tananbaum, H., Avni, Y., Branduardi, G., et al. 1979, ApJ, 234, L9

Terashima, Y., \& Wilson, A. S. 2003, ApJ, 583, 145

Terlevich, R., \& Melnick, J. 1985, MNRAS, 213, 841

Trussoni, E., Vagnetti, F., Massaglia, S., et al. 1999, A\&A, 348, 437

Turner, M. J., Abbey, A., Arnaud, M., et al. 2001, A\&A, 365, L27

Vasudevan, R. V., \& Fabian, A. C. 2007, MNRAS, 381, 1235

Venturi, T., Morganti, R., Tzioumis, T., \& Reynolds, J. 2000, EVN Symposium 2000, Proceedings of the 5th european VLBI Network Symposium, 47

Wu, Q., Yuan, F., \& Cao, X. 2007, ApJ, in press [arXiv: 0706.4124]

Yuan, F., \& Cui, W. 2005, ApJ, 629, 408

Zdziarski, A. A., Lubinski, P., Gilfanov, M., \& Revnivtsev, M. 2003, MNRAS, 342,355

Zombeck, M. V. 1990, Handbook of Space Astronomy and Astrophysics (Cambridge: Cambridge Univ. Press) 